AEI-2002-072

hep-th/0209157

\title{
On the hierarchy of symmetry breaking D-branes in group manifolds
}

\author{
Thomas Quella* \\ Max-Planck-Institut für Gravitationsphysik \\ (Albert-Einstein-Institut) \\ Am Mühlenberg 1 \\ D-14476 Golm \\ Germany
}

September 19, 2002

\begin{abstract}
We construct the boundary WZNW functional for symmetry breaking D-branes on a group manifold which are localized along a product of a number of twisted conjugacy classes and which preserve an action of an arbitrary continuous subgroup. These branes provide a geometric interpretation for the algebraic formulation of constructing D-branes developed recently in hep-th/0203161. We apply our results to obtain new symmetry breaking and non-factorizing D-branes in the background $S L(2, \mathbb{R}) \times S U(2)$.
\end{abstract}

\section{Introduction}

The classification of D-branes in given string backgrounds is one of the most important tasks in string theory. The interest is mainly based on the occurence of non-perturbative dualities between different types of string theories and on the natural appearance of (non-commutative) gauge theories in the low energy description of open strings. The classification of D-branes relies on finding conformally invariant boundary conditions for a given two-dimensional bulk conformal field theory (CFT). Presently one of the best understood classes of CFT's is provided by the Wess-Zumino-Novikov-Witten (WZNW) theories [1], 2] which describe string theory on group manifolds [3]. Although for dimensional reasons most groups cannot be part of a consistent string background, they constitute an important ingredient in model building via coset constructions [ [⿴囗口.

*E-mail: quella@aei.mpg.de 
In the last few years we have made significant progress with the classification of Dbranes in a group manifold $G$. For a long time only two types have been known: untwisted [5] and twisted D-branes [6, [7. Both share the property of preserving the maximal possible symmetry. By now we have an almost complete understanding of these D-branes. In particular, their geometry was shown to be given by twisted conjugacy classes [8, 9]. This observation was supported by a Lagrangian description [10] and by a Born-Infeld analysis which also established the stability of these D-branes [11, 12]. Finally, the discovery of the noncommutative geometry associated to these branes [13, 14 allowed the discussion of their dynamics 15, 16, 14.

In contrast to these successes in the study of maximally symmetric D-branes only recently methods have been developed to deal with D-branes which break part of the symmetry 17, 18, 19, 20]. In the work of Maldacena, Moore and Seiberg this was achieved by using a certain kind of T-duality related to $U(1)$ subgroups [17, 18]. A conceptually different and more general algebraic framework which also incorporates the usage of nonabelian subgroups was developed in [20]. While the geometric interpretation in the first case is obvious from T-duality, a geometric interpretation for more general symmetry breaking D-branes was missing until now. In the present work we partly fill this gap and show that the most natural symmetry breaking D-branes in group manifolds which preserve a given continuous subgroup $H \hookrightarrow G$ are localized along products of quantized twisted conjugacy classes of subgroups $U_{l}$ where $H=U_{1} \hookrightarrow U_{2} \cdots \hookrightarrow U_{n}=G$. We will prove this fact by constructing the corresponding boundary WZNW functional. The identification with D-branes obtained from the algebraic description [20] is established by evaluating the closed string couplings to the branes. It is further supported by an analysis of the spectrum of open strings arising from both approaches. This comparison relies on the noncommutative geometry associated to twisted D-branes [13, 14]. For completeness we should add that a Lagrangian description of symmetry breaking D-branes which are accessible by T-duality has already been found in [21]. These, however, are based on the inclusion $U(1) \hookrightarrow G$ and thus constitute only a small subset of an enormous hierarchy of symmetry breaking D-branes arising from embedding chains of non-abelian subgroups 20 .

We apply our geometric construction to the classification of D-branes in the background $S L(2, \mathbb{R}) \times S U(2)$. A large number of symmetry breaking and non-factorizing D-branes is revealed which preserve a continuous subgroup of the target space. All these branes may easily be lifted to the string background $A d S_{3} \times S^{3} \times T^{4}$ by considering the covering space $A d S_{3}$ of $S L(2, \mathbb{R})$ and using the equivalence $S U(2) \cong S^{3}$. D-branes in the individual factors $S L(2, \mathbb{R})$ and $S U(2)$ have been described before in [22, 23, 17, 24, 21] for instance. Our results confirm the geometric description of symmetry breaking D-branes in these groups which has been obtained using T-duality.

This paper is organized as follows. In the next section we will construct the boundary WZNW functional for symmetry breaking D-branes which are localized along the product 
of twisted conjugacy classes of subgroups $U_{l}$ which are organized in an embedding chain $H=U_{1} \hookrightarrow U_{2} \cdots \hookrightarrow U_{n}=G$. We will argue in section 2 that these D-branes correspond to symmetry breaking boundary states which have been constructed in [20] using algebraic methods. Our arguments are based on a target space reinterpretation. In the "new" target space the D-branes factorize and the coupling to closed strings as well as the spectrum of open strings can easily be determined. We find full agreement with the Lagrangian approach. The general geometric description for symmetry breaking D-branes is applied to the classification of D-branes in the group manifold $S L(2, \mathbb{R}) \times S U(2)$ in section 4 . Finally, we conclude with open problems and further related topics to study.

\section{The Lagrangian approach}

In conformal field theory language, WZNW models are based on a chiral algebra $\mathcal{A}(G)$ which is generated by an affine Kac-Moody algebra $\hat{\mathfrak{g}}_{k}$ at level $k$. The algebra $\hat{\mathfrak{g}}_{k}$ is the affine extension of the Lie algebra $\mathfrak{g}$ belonging to the group manifold $G$, the target space under consideration. The bulk symmetry is given by two independent copies $\mathcal{A}(G) \oplus \overline{\mathcal{A}(G)}$ of the chiral algebra. On the boundary holomorphic and antiholomorphic degrees of freedom are coupled, and at most one copy of $\mathcal{A}(G)$ can be preserved. It is natural to consider D-branes which preserve only a given subsymmetry $\mathcal{A}(H) \hookrightarrow \mathcal{A}(G)$. Here, $\mathcal{A}(H)$ is the chiral algebra generated by an affine Kac-Moody subalgebra $\hat{\mathfrak{h}}_{k^{\prime}} \hookrightarrow \hat{\mathfrak{g}}_{k}$. All such embeddings arise from continuous subgroups $H$ of $G$.

The boundary theory immediately becomes non-rational if one tries to preserve only the symmetry $\mathcal{A}(H)$ on the boundary. Following [20] it is thus natural to consider an embedding chain of subgroups"

$$
H=U_{1} \hookrightarrow U_{2} \hookrightarrow \cdots \hookrightarrow U_{n-1} \hookrightarrow U_{n}=G
$$

and to preserve instead the larger chiral algebra

$$
\mathcal{A}\left(U_{1}\right) \oplus \mathcal{A}\left(U_{2} / U_{1}\right) \oplus \cdots \oplus \mathcal{A}\left(U_{n} / U_{n-1}\right) \hookrightarrow \mathcal{A}(G)
$$

which is rational with respect to the original theory. In writing down this expression we denoted by $\mathcal{A}\left(U_{l+1} / U_{l}\right)$ the coset chiral algebras which arise via GKO construction 4 . By a straightforward generalization of [20] we may construct D-branes which preserve the chiral algebra (2.2) and calculate their spectrum of open strings. The geometric interpretation of this kind of D-branes, however, remained obscure up to now. The decomposition (2.2) only implies that the submanifolds of $G$ in which these D-branes are localized should admit an action of the subgroup $H$.

\footnotetext{
${ }^{1} \mathrm{We}$ will use the phrase embedding map to denote a group homomorphism which descends to an injective map on the level of the Lie algebras. This condition ensures that the embedding map preserves the dimension, but allows for non-trivial wrapping numbers for instance.
} 
In this article we provide the missing link between algebra and geometry. The remaining part of this section will be devoted to the construction of a boundary WZNW functional for D-branes which are localized along the product of a number of twisted conjugacy classes, one for each subgroup $U_{l}$. These submanifolds of $G$ admit an action of $H$ which is inherited from the embedding chain (2.1). The WZNW functional will be shown to be invariant under this action. These D-branes thus provide natural candidates for being geometric counterparts of those arising from the general algebraic framework [20]. In the next section we will give additional arguments to support this claim. This includes the calculation of closed string couplings to the branes and an analysis of the spectrum of open strings.

Let us be a bit more precise about what we mean by "product of a number of twisted conjugacy classes". For an exact treatment we have to specify embeddings of the individual subgroups $U_{l}$ into $G$ and an action of $H$ on each of these subgroups. Assume that we have given embeddings $\epsilon^{U_{l} U_{l+1}}$ of $U_{l}$ in $U_{l+1}$ which specify the embedding chain (2.1). In addition we will assume the existence of - possibly trivial - automorphisms $\Omega_{l}$ of $U_{l}$. This information may be used to define embeddings

$$
\begin{aligned}
& \epsilon^{H U_{l}}=\epsilon^{U_{l-1} U_{l}} \circ \cdots \circ \epsilon^{U_{2} U_{3}} \circ \epsilon^{U_{1} U_{2}} \\
& \epsilon_{\Omega}^{U_{l} G}=\Omega_{n} \circ \epsilon^{U_{n-1} U_{n}} \circ \Omega_{n-1} \circ \cdots \circ \epsilon^{U_{l+1} U_{l+2}} \circ \Omega_{l+1} \circ \epsilon^{U_{l} U_{l+1}} .
\end{aligned}
$$

The embedding $\epsilon_{\Omega}^{U_{l} G}$ contains the automorphisms $\Omega_{k}$ at every stage of the embedding chain except for the starting point $U_{l}$. The geometry of our D-branes is the following product of images of twisted conjugacy classes,

$$
\mathcal{D}\left\{U_{l}, \Omega_{l}, f_{l}\right\}=\mathcal{C}_{f_{n}}^{U_{n}}\left(\Omega_{n}\right) \cdot \epsilon_{\Omega}^{U_{n-1} G}\left(\mathcal{C}_{f_{n-1}}^{U_{n-1}}\left(\Omega_{n-1}\right)\right) \cdot \ldots \cdot \epsilon_{\Omega}^{U_{1} G}\left(\mathcal{C}_{f_{1}}^{U_{1}}\left(\Omega_{1}\right)\right) .
$$

The labels $f_{l} \in U_{l}$ have to satisfy certain quantization conditions and constraints which arise from branching selection rules associated to the embedding chain (2.1). A discussion of these issues follows below. The exact definition of the twisted conjugacy classes is given by

$$
\mathcal{C}_{f_{l}}^{U_{l}}\left(\Omega_{l}\right)=\left\{s_{l} f_{l} \Omega_{l}\left(s_{l}^{-1}\right) \mid s_{l} \in U_{l}\right\}
$$

Let us denote by $c_{l}$ elements of the image of twisted conjugacy classes in $G$, i.e. $c_{l} \in$ $\epsilon_{\Omega}^{U_{l} G}\left(\mathcal{C}_{f_{l}}^{U_{l}}\left(\Omega_{l}\right)\right)$. Each of these conjugacy classes admits an action of $H$ which can be formulated as follows,

$$
s_{l} \mapsto \epsilon^{H U_{l}}(h) \cdot s_{l} \quad \Rightarrow \quad c_{l} \mapsto \epsilon_{\Omega}^{U_{l} G} \circ \epsilon^{H U_{l}}(h) \cdot c_{l} \cdot \epsilon_{\Omega}^{U_{l} G} \circ \Omega_{l} \circ \epsilon^{H U_{l}}\left(h^{-1}\right) .
$$

Due to the recursion relations $\epsilon_{\Omega}^{U_{l+1} G} \circ \Omega_{l+1} \circ \epsilon^{U_{l} U_{l+1}}=\epsilon_{\Omega}^{U_{l} G}$ also the product of twisted conjugacy classes (2.4) admits a well-defined action of $H$. An arbitrary element $x \in$ $\mathcal{D}\left\{U_{l}, \Omega_{l}, f_{l}\right\}$ transforms as

$$
x \mapsto \epsilon^{H U_{n}}(h) \cdot x \cdot \epsilon_{\Omega}^{U_{1} G} \circ \Omega_{1}\left(h^{-1}\right) \in \mathcal{D}\left\{U_{l}, \Omega_{l}, f_{l}\right\} .
$$


As conjectured above, the subset $\mathcal{D}\left\{U_{l}, \Omega_{l}, f_{l}\right\}$ of $G$ thus indeed provides a natural candidate for a geometric description of the symmetry breaking D-branes which arise from the algebraic description via the decomposition (2.2). Notice that our results obviously reduce to the usual description of maximally symmetric D-branes by taking $\Omega_{l}=$ id and $f_{l}=e$ for $l<n$. It is remarkable that the notion of twist becomes much richer for symmetry breaking D-branes in comparison to maximally symmetric ones. We are indeed allowed to take non-trivial twists for each of the subgroups $U_{l}$.

We are now prepared to substantiate our conjecture by constructing a boundary WZNW functional for D-branes localized along the subset (2.4) which is invariant under the action (2.5) of $H$. Geometrically, the theory is described by a non-linear $\sigma$-model of fields $g: \Sigma \rightarrow G$ which live on a two-dimensional world sheet $\Sigma$ and which take values in the group manifold $G$. We will always assume that $G$ is simple in what follows, but it is straightforward to generalize our results to reductive groups, i.e. to those which are a direct product of simple groups and $U(1)$ factors. The action functional for this theory is given by

$$
S_{\mathrm{WZNW}}^{G}(g ; k \mid \mathcal{D})=S_{\mathrm{kin}}^{G}(g ; k)+S_{\mathrm{WZ}}^{G}(g ; k)+S_{\mathcal{D}}^{G}(g ; k)
$$

and consists of three parts, the usual kinetic term, the so-called Wess-Zumino term and a boundary term. All of them contain a parameter $k>0$ subject to certain consistency conditions (see below). The kinetic term is given by

$$
S_{\text {kin }}^{G}(g ; k)=-\frac{k}{4 \pi} \frac{2}{I_{R}} \int_{\Sigma} d^{2} z \operatorname{tr}_{R}\left\{\partial g g^{-1} \bar{\partial} g g^{-1}\right\} .
$$

The trace is evaluated in some non-trivial unitary representation $R$ of the Lie algebra $\mathfrak{g}$ of $G$. This is indicated by the explicit appearance of the Dynkin index $I_{R}$ of the representation. The symbol $\operatorname{tr}_{R}$ denotes the trace of $\operatorname{dim} R$-dimensional matrices. The combination $2 / I_{R} \operatorname{tr}_{R}$ is a normalized trace which is independent of the representation $R$. In our conventions the Killing form is obtained from $I_{R} \kappa^{\alpha \beta}=\operatorname{tr}_{R}\left\{R\left(T^{\alpha}\right) R\left(T^{\beta}\right)\right\}$.

The Wess-Zumino term is defined in terms of its associated 3-form $\omega^{\mathrm{WZ}}$. Its contribution to the boundary WZNW functional (2.7) is given by

$$
S_{\mathrm{WZ}}^{G}(g ; k)=-\frac{k}{4 \pi} \frac{2}{I_{R}} \int_{B} \omega^{\mathrm{WZ}} \quad \text { with } \quad \omega^{\mathrm{WZ}}(g)=\frac{1}{3} \operatorname{tr}_{R}\left(g^{-1} d g\right)^{3} .
$$

This integral extends over a three-dimensional manifold $B$ whose boundary is given by $\partial B=\Sigma \cup D$ where $D$ is a disjoint union of (topological) discs filling the holes of $\Sigma$ such that $\Sigma \cup D$ has no boundaries. For notational simplicity we will assume that $D$ consists of exactly one disc.

If one only considers the first two terms of the action functional (2.7), it is invariant under all transformations $g \mapsto g_{L}(z) g g_{R}^{-1}(\bar{z})$. This is the famous loop group symmetry $G(z) \times G(\bar{z})$ of the WZNW theory. This symmetry is generated by two Lie algebra valued 
currents $J(z)=-k \partial g g^{-1}$ and $\bar{J}(\bar{z})=k g^{-1} \bar{\partial} g$ which are chiral by the equations of motion. They generate two commuting copies of the affine Kac-Moody algebra $\hat{\mathfrak{g}}_{k}$ at level $k$ as mentioned already in the beginning of this section.

To complete the definition of the boundary WZNW functional (2.7) we finally have to define the boundary term. This is given by the integral

$$
S_{\mathcal{D}}^{G}(g ; k)=\frac{k}{4 \pi} \frac{2}{I_{R}} \int_{D} \omega_{\mathcal{D}}=\frac{k}{4 \pi} \frac{2}{I_{R}} \int_{D} \sum_{l=1}^{n} \sum_{k=1}^{l} \omega_{\mathcal{D}}\left(c_{k}, \cdots, c_{l}\right)
$$

over the auxiliary disc $D$. We assume that the boundary of $\Sigma$ and the whole disc $D$ are mapped into the subset $\mathcal{D}\left\{U_{l}, \Omega_{l}, f_{l}\right\}$ of $G$. The first condition justifies the use of the word D-brane when referring to this submanifold. The boundary two-forms entering the definition (2.8) are specified by

$$
\begin{aligned}
\omega_{\mathcal{D}}\left(c_{l}\right) & =\operatorname{tr}_{R}\left\{\epsilon^{U_{l} G}\left(s_{l}^{-1} d s_{l} f_{l} \Omega_{l}\left(s_{l}^{-1} d s_{l}\right) f_{l}^{-1}\right)\right\} \\
\omega_{\mathcal{D}}\left(c_{k}, \cdots, c_{l}\right) & =-\operatorname{tr}_{R}\left\{c_{k}^{-1} \cdots c_{l}^{-1} d c_{l} c_{l-1} \cdots c_{k+1} d c_{k}\right\} .
\end{aligned}
$$

If $\mathcal{D}\left\{U_{l}, \Omega_{l}, f_{l}\right\}$ is a single twisted conjugacy class, the expression (2.8) reduces to those found for maximally symmetric D-branes [10]. For a product of two twisted conjugacy classes we recover boundary terms which have been used to describe maximally symmetric D-branes in coset spaces [25, 26]. Our expressions also contain as a special case the recent results of [21] by taking the "embedding chain" $U(1) \hookrightarrow G$ and a particular choice of automorphisms. The exact correspondence will be subject of section 4 .

After having provided the complete definition of the boundary WZNW functional a few remarks are in order. The physics which is described by the action (2.7) should not depend on the two auxiliary manifolds $B$ and $D$. This leads to restrictions such as quantization conditions for the level and allowed conjugacy classes as well as branching selection rules 10, 25, 26. Let us be a little bit more specific. For compact simply-connected simple Lie groups topological considerations regarding the Wess-Zumino term force $k$ to be a positive integer. This may be different for non-simply-connected or non-compact groups. In the case of $G=S O(3)$ the level $k$ has to be even for instance and for $G=S L(2, \mathbb{R})$ we obtain no additional constraints on the level. Invariance of the action functional (2.7) under infinitesimal deformations of the disc is ensured by the relation $d \omega_{\mathcal{D}}=\left.\omega^{\mathrm{WZ}}\right|_{\mathcal{D}}$ which is proven in the appendix. Taking global aspects of the embedding of the disc into account one exactly recovers the quantization of twisted conjugacy classes and branching selection rules which would be expected from the CFT description [10, 25, 26]. This concludes our arguments that the action (2.7) is well-defined.

As was discussed in [25, 26] the boundary two-form $\omega_{\mathcal{D}}$ does not only depend on the values of the field $g$ on the boundary but also on the exact decomposition into a product $g=c_{n} \cdots c_{1}$ of elements of the individual twisted conjugacy classes. Under certain circumstances the sets $\mathcal{D}\left\{U_{l}, \Omega_{l}, f_{l}\right\}$ and $\mathcal{D}\left\{U_{l}^{\prime}, \Omega_{l}^{\prime}, f_{l}^{\prime}\right\}$ are identical. The algebraic 
analysis, however, suggests that they should describe different D-branes with different spectrum and different mass density. This becomes particularly important for products of twisted conjugacy classes which cover the whole group $G$. The solution to this puzzle is presented in section 3.1 where it is shown that it is natural to adopt a different target space interpretation when using the decomposition (2.2). In this larger target space the shape of all our D-branes is distinct.

It remains to be shown that the boundary WZNW functional (2.7) is invariant under the action $(2.5,2.6)$ of $H$ on the boundary. To be specific, the symmetry $G(z) \times G(\bar{z})$ of the bulk theory has to be broken to a symmetry $H(\tau) \hookrightarrow G(\tau) \times G(\tau)$ on the boundary where the embedding is given by $\left(\epsilon^{H U_{n}}, \epsilon_{\Omega}^{U_{1} G_{\circ}} \Omega_{1}\right)$. With the same embedding map one can define an action of $H(z) \times H(\bar{z})$ in the bulk theory which induces the decomposition (2.2) of chiral algebras. This reduced action gives rise to chiral currents $J^{\mathfrak{h}}(z)$ and $\bar{J}^{\mathfrak{h}}(\bar{z})$ which take values in the Lie algebra $\mathfrak{h}$. Demanding that this action reduces to the action of $H(\tau)$ on the boundary is equivalent to enforcing trivial gluing conditions on the currents $J^{\mathfrak{h}}(z)$ and $\bar{J}^{\mathfrak{h}}(\bar{z})$. Note that it is not relevant whether one demands twisted gluing conditions or puts the twist in the definition of the current as the decomposition of the bulk Hilbert space also has to reflect this choice. In our previous considerations we adopted the second point of view.

Let us now determine the variation of the boundary WZNW functional under an arbitrary infinitesimal action of $h(\tau)=1+i \omega(\tau) \in H(\tau)$. A lengthy but straightforward calculation results in

$$
\delta \omega_{\mathcal{D}}=-i \sum_{l=1}^{n} \operatorname{tr}\left\{d \omega_{L}^{(n)} c_{n} \cdots c_{l+1} d c_{l} c_{l}^{-1} \cdots c_{n}^{-1}+d \omega_{R}^{(1)} c_{1}^{-1} \cdots c_{l}^{-1} d c_{l} c_{l-1} \cdots c_{1}\right\}
$$

where we introduced the abbreviations $\omega_{L}^{(n)}=\epsilon^{H U_{n}}(\omega)$ and $\omega_{R}^{(1)}=\epsilon_{\Omega}^{U_{1} G} \circ \Omega_{1}(\omega)$. The variation of the Wess-Zumino term may be determined from

$$
\delta \omega^{\mathrm{WZ}}=-i d \operatorname{tr}\left\{d \omega_{L}^{(n)} d g g^{-1}+d \omega_{R}^{(1)} g^{-1} d g\right\}
$$

After integration it will give two contributions which arise from the boundary $\Sigma \cup D$ of $B$. The first one belonging to $\Sigma$ is canceled by the variation of the kinetic term. If we restrict the discussion to the disc which is mapped to the set $\mathcal{D}\left\{U_{l}, \Omega_{l}, f_{l}\right\}$, the variation (2.10) further simplifies to

$$
\left.\delta \omega^{\mathrm{WZ}}\right|_{D}=-i \sum_{l=1}^{n} d \operatorname{tr}\left\{d \omega_{L}^{(n)} c_{n} \cdots c_{l+1} d c_{l} c_{l}^{-1} \cdots c_{n}^{-1}+d \omega_{R}^{(1)} c_{1}^{-1} \cdots c_{l}^{-1} d c_{l} c_{l-1} \cdots c_{1}\right\}
$$

Obviously, the contributions from $\delta \omega^{\mathrm{WZ}}$ and $\delta \omega_{\mathcal{D}}$ cancel each other exactly. The details of the calculation can be found in the appendix. This completes the proof of the symmetry of the D-branes $\mathcal{D}\left\{U_{l}, \Omega_{l}, f_{l}\right\}$ under the action of the subgroup $H$. 
The procedure described in this section provides a whole hierarchy of symmetry breaking D-branes. The classification of all these objects is greatly simplified by the following observation which is well-known from maximally symmetric D-branes. Instead of allowing all choices of automorphisms we may restrict to the case of outer automorphisms. The appearance of an inner automorphism $\Omega_{l}\left(u_{l}\right)=b_{l} u_{l} b_{l}^{-1}$ in the product of twisted conjugacy classes (2.4) just corresponds to putting this automorphism to the identity in the expressions (2.3) and (2.4), to replace $f_{l}$ by $f_{l} b_{l}$ and to multiply the resulting expression for eq. (2.4) with the element $\epsilon_{\Omega}^{U_{l} G}\left(b_{l}^{-1}\right)$ from the right. Geometrically, this procedure induces an overall shift. The same idea also enables us to choose specific representatives for whole classes of outer automorphisms by separating their "inner part".

One may ask whether arranging the twisted conjugacy classes in the definition (2.4) in different order would lead to new results. It is easy to show that this is not the case. Exchanging two conjugacy classes merely leads to a redefinition of embedding maps and automorphisms. Under these circumstances it is natural to work with one standard representative. In our case the latter is defined to be given by eq. (2.4).

We conclude with a few remarks. The first concerns the dimension of the D-branes which correspond to the product of twisted conjugacy classes (2.4). For a naive evaluation of the dimension one would simply add the dimensions of the twisted conjugacy classes present in eq. (2.4). It is obvious that this procedure would rapidly exceed the dimension of the group itself if one takes embedding chains (2.1) with a large number of subgroups. Up to now we lack a general dimension formula for this kind of D-branes. Let us emphasize at this point the remarkable fact that they tend to be more and more space-filling the more of the symmetry we break. We believe that branes which cover the whole target space can be constructed for every WZNW model. A natural candidate for such a space-filling brane is obtained by taking the product of a non-degenerate ordinary conjugacy class of $G$ and a distinguished twisted conjugacy class of its maximal torus $T=U(1)^{\operatorname{rank} G}$. The first is isomorphic to $G / T$ [9], i.e. has $\operatorname{dimension} \operatorname{dim} G-\operatorname{rank} G$, while the second is given by $T$ itself. In section 1 we will confirm our conjecture in a number of examples.

The second remark concerns the hierarchical structure of the symmetry breaking Dbranes which are described by the product of twisted conjugacy classes (2.4). Let us consider a fixed embedding chain (2.1). By choosing an automorphism $\Omega_{l}$ to act trivially and the corresponding element $f_{l}$ to be given by the group unit we can achieve that the conjugacy class $\mathcal{C}_{f_{l}}^{U_{l}}\left(\Omega_{l}\right)$ may be omitted from the expression (2.4) for the geometry of the D-brane. This means that we could have equally well omitted the group $U_{l}$ from the embedding chain (2.1) in order to describe the same D-brane. The same feature has also been observed in the algebraic description [20]. To obtain a classification of D-branes in the group manifold $G$ which preserve an arbitrary continuous subgroup it is thus enough to find all inequivalent chains of maximal embeddings. 


\section{The algebraic point of view}

In the last section we used the Lagrangian approach to construct a large number of Dbranes on a group manifold $G$ which preserve a given continuous subgroup $H$. We also presented first indications that these provide the geometric interpretation for symmetry breaking boundary states arising in the algebraic approach [20]. In the following we will further illuminate this equivalence. Full agreement is found when calculating the coupling of closed strings to the branes. Also the spectrum of open strings which is predicted by geometry fits into the algebraic description. Before we dive into the discussion of branes we present a natural target space reinterpretation which sheds new light on some open issues which have been the cause of some confusion in the past. As no general tools are available for an algebraic description of non-compact WZNW theories, we assume the group $G$ to be compact in what follows. To simplify notation the group is also considered to be simply-connected and simple.

\subsection{Target space reinterpretation}

The usual interpretation of a WZNW theory relies on the group $G$ itself as target space. In the context of the decomposition (2.2) of the chiral algebra it is, however, more convenient to work with the space

$$
G^{\text {new }}=\frac{U_{n} \times U_{n-1} \times U_{n-1} \times \cdots \times U_{1} \times U_{1}}{U_{n-1} \times U_{n-1} \times \cdots \times U_{1} \times U_{1}}=\frac{G \times X}{X} .
$$

This statement is a generalization of a proposal which has been formulated in the context of coset theories [27]. The specific form of the auxiliary space $X$ has its origin in the decomposition of chiral algebras (2.2). It is motivated by the deep relation between coset CFT's $U_{l+1} / U_{l}$ and product CFT's $U_{l+1} \times U_{l}$ which itself is based on the similarity of modular properties. The extension of $G$ by $X$ introduces additional degrees of freedom which have to be removed by dividing through $X$. The exact action of $X$ will be given in eq. (3.2). We will argue below that the product of twisted conjugacy classes (2.4) which has been defined on $G$ possesses a natural interpretation as a direct product in the numerator $G \times X$ of the new target space $G^{\text {new }}$. In this picture each twisted conjugacy class of a group $U_{l}$ with $l<n$ is diagonally embedded in the product $U_{l} \times U_{l}$.

The equivalence of the spaces $G$ and $G^{\text {new }}$ seems to be obvious at first sight. Nevertheless we have to be very careful as $G$ carries additional structure which should be reflected in $G^{\text {new }}$. In particular, $G$ admits an action of the group $G \times G$, i.e. the regular action from the left and from the right. The group $G \times G$ should be considered as the "constant" part of the WZNW symmetry $G(z) \times G(\bar{z})$ which has been described in the last section. When we consider symmetry breaking D-branes which arise from the embedding chain (2.1), the action of $G \times G$ thus has to be broken to an action of the subgroup $H \times H$ where the embedding of the latter is given by the map $\left(\epsilon^{H U_{n}}, \epsilon_{\Omega}^{U_{1} G} \circ \Omega_{1}\right)$. We will argue below that 
the same action of $H \times H$ can be found on $G^{\text {new }}$ provided that one uses the correct action of $X$ on $G \times X$ in the definition (3.1). To be precise, the elements of $G^{\text {new }}$ should be given by tupels $\left(u_{n}, u_{n-1}^{\prime}, u_{n-1}, \cdots, u_{2}^{\prime}, u_{2}, u_{1}^{\prime}, u_{1}\right) \in G \times X$ subject to the identifications

$$
\left(u_{l}^{\prime}, u_{l}\right) \sim\left(u_{l}^{\prime} \cdot t_{l}^{-1}, t_{l} \cdot u_{l}\right) \quad \text { and } \quad\left(u_{l+1}, u_{l}^{\prime}\right) \sim\left(u_{l+1} \cdot \Omega_{l+1} \circ \epsilon^{U_{l} U_{l+1}}\left(s_{l}^{-1}\right), s_{l} \cdot u_{l}^{\prime}\right)
$$

for $t_{l}, s_{l} \in U_{l}$. The action of $H \times H$ on the target space $G^{\text {new }}$ on the other hand should be defined by $\left(u_{n}, \cdots, u_{1}\right) \mapsto\left(\epsilon^{H U_{n}}\left(h_{1}\right) u_{n}, \cdots, u_{1} \Omega_{1}\left(h_{2}^{-1}\right)\right)$. The identification (3.2) shows that the new target space $G^{\text {new }}$ is a specific example of an asymmetric coset. A general discussion of string theory in asymmetric coset spaces will follow in [28].

The connection of the new target space $G^{\text {new }}$ to the considerations in the previous section may easily be illustrated by working out the natural representatives of elements in $G^{\text {new }}$. They are given by

$$
\left(u_{n} \cdot \epsilon_{\Omega}^{U_{n-1} G}\left(u_{n-1}^{\prime} \cdot u_{n-1}\right) \cdot \ldots \cdot \epsilon_{\Omega}^{U_{1} G}\left(u_{1}^{\prime} \cdot u_{1}\right), e, \ldots, e\right) .
$$

A comparison with eq. (2.4) indicates that the product of twisted conjugacy classes which appeared in the last section has a natural interpretation in the new target space $G^{\text {new }}$. The relation (3.3) indeed shows that elements of $G^{\text {new }}$ may be represented naturally as elements of $G$. Note however that one and the same element of $G$ is represented by a whole orbit of elements in $G \times X$. This makes explicit the drastical increase of degrees of freedom which are associated to the decomposition (2.2) of the chiral algebra. What happened to be a cause of confusion in [25, 26] and in the previous section, has now found its natural explanation. On the contrary, it seems to give us the possibility to describe new interesting features such as superpositions of D-branes and multiple wrappings (see also [17]).

The reader may wonder why we had to choose such a complicated identification (3.2) to define the coset $G^{\text {new }}$. A partial answer was given already by the striking relation between the representative (3.3) and the form of the product of twisted conjugacy classes (2.4). The deeper reason for this particular choice of identification comes, however, from demanding the equality of $G$ and $G^{\text {new }}$ including the given action of $H \times H$ on them. The latter can be understood best if one does not work on the level of manifolds but descends to the algebras of functions $\mathcal{F}(G)$ and $\mathcal{F}\left(G^{\text {new }}\right)$ which inherit the given action of $H \times H$ but allow for a linear representation. According to a theorem of Gel'fand and Naimark also the topology of a manifold is completely contained in its algebra of functions. Showing the equality

$$
\mathcal{F}(G) \cong \mathcal{F}\left(G^{\text {new }}\right)=\operatorname{Inv}_{X}(\mathcal{F}(G \times X))
$$

as $H \times H$ modules is thus enough to establish the equivalence of the target spaces $G$ and $G^{\text {new }}$ including the action of $H \times H$ on them. The relation (3.4) may be proven by using the Peter-Weyl theorem which gives the decomposition of the algebra of functions on a 
group into irreducible representations under left and right regular action of the group itself. Restricting the action to $H \times H$ and taking all twists into account we exactly recover the equality (3.4).

The considerations of the last paragraph have direct implications for the conformal field theory description. Let us bring to mind that the interpretation of $G$ as the target space of a WZNW theory is supported by the deep relation between the spectrum of closed strings and the algebra of functions on the group. According to the Peter-Weyl theorem the algebra of functions $\mathcal{F}(G)$ is recovered from the ground state structure of the charge conjugate partition function of the WZNW theory in the limit $k \rightarrow \infty$ when interpreted as a $G \times G$ module with respect to left and right regular action of $G$. As already mentioned above, the group $G \times G$ should be considered as the "constant" part of $G(z) \times G(\bar{z})$. After symmetry reduction, the decomposition (2.2) of the chiral algebras has to be accompanied by an analogous decomposition of the closed string Hilbert space. On the geometrical side this corresponds to the interpretation of the $G \times G$ module $\mathcal{F}(G)$ as an $H \times H$ module where the embedding is given by $\left(\epsilon^{H U_{n}}, \epsilon_{\Omega}^{U_{1} G} \circ \Omega_{1}\right)$.

\subsection{The coupling of boundary states to closed strings}

The main aim of this section is to understand the relation between the geometric results of section 2 and the algebraic method of constructing symmetry breaking boundary states [20]. It is thus necessary to recapitulate the main formulas of this approach. We will be rather sketchy in what follows. In particular we will not be concerned with technical difficulties such as field identification or related topics. We will tacitly assume that this

phenomenon does not appear for the cases under consideration. In the geometric regime where the level $k$ runs to infinity, this seems to be a valid approximation in most of the cases. The interested reader is referred to [20] where he can find the missing details. The general framework of boundary conformal field theory is subject of [29, 30, 31, 32, 17, 33] for instance.

The decomposition of the chiral algebras (2.2) is accompanied by a decomposition

$$
\mathcal{H}_{\mu_{n}}^{G}=\bigoplus \mathcal{H}_{\left(\mu_{n}, \mu_{n-1}\right)}^{U_{n} / U_{n-1}} \otimes \cdots \otimes \mathcal{H}_{\left(\mu_{2}, \mu_{1}\right)}^{U_{2} / U_{1}} \otimes \mathcal{H}_{\mu_{1}}^{U_{1}}
$$

of representation spaces $\mathcal{H}_{\mu_{n}}^{G}$ of $\mathcal{A}(G)$. It is very important, however, that the decomposition of the antiholomorphic part looks slightly different as it has to reflect the different choices of $H$-actions which are used to define the currents $J^{\mathfrak{h}}(z)$ and $\bar{J}^{\mathfrak{h}}(\bar{z})$. To be precise we obtain

$$
\overline{\mathcal{H}}_{\mu_{n}}^{G}=\bigoplus \overline{\mathcal{H}}_{\left(\Omega_{n}^{-1}\left(\mu_{n}\right), \nu_{n-1}\right)}^{U_{n} / U_{n-1}} \otimes \cdots \otimes \overline{\mathcal{H}}_{\left(\Omega_{2}^{-1}\left(\nu_{2}\right), \nu_{1}\right)}^{U_{2} / U_{1}} \otimes \overline{\mathcal{H}}_{\Omega_{1}^{-1}\left(\nu_{1}\right)}^{U_{1}}
$$

We observe that the weights $\nu_{l}$ appear with a relative twist in the different representation spaces. The last two relations induce an analogous decomposition of the full charge conjugate closed string Hilbert space $\mathcal{H}^{G}$. 
Before we are able to construct the boundary states which contain all the relevant data to perform the comparison with the geometric picture we need some further preparations. Assume that we have given the algebraic solution corresponding to twisted gluing conditions in the auxiliary algebras $\mathcal{A}\left(U_{l}\right)$. This means that we know structure constants $\psi^{U_{l}}$ such that the matrices

$$
\left(n_{\nu_{l}}^{U_{l}}\right)_{\rho_{l}}^{\rho_{l}^{\prime}}=\sum_{\Omega_{l}\left(\mu_{l}\right)=\mu_{l}} \frac{\left(\bar{\psi}^{U_{l}}\right)_{\rho_{l}^{\prime}}^{\mu_{l}}\left(\psi^{U_{l}}\right)_{\rho_{l}}^{\mu_{l}} S_{\mu_{l} \nu_{l}}^{U_{l}}}{S_{\mu_{l} 0}^{U_{l}}}
$$

form a NIM-rep of the fusion algebra of the affine Kac-Moody algebra $\left(\hat{\mathfrak{u}}_{l}\right)_{k_{l}}$. In this expression we denoted by $S^{U_{l}}$ the modular S matrix of $\left(\hat{\mathfrak{u}}_{l}\right)_{k_{l}}$. The structure constants $\left(\psi^{U_{l}}\right)_{\rho_{l}}^{\mu_{l}}$ carry two labels. One of them refers to boundary conditions $\rho_{l}$ while the other refers to symmetric representations $\mu_{l}=\Omega_{l}\left(\mu_{l}\right)$ of $\left(\hat{\mathfrak{u}}_{l}\right)_{k_{l}}$. The simplest example of a NIMrep may be obtained from $\Omega_{l}=$ id by putting $\left(\psi^{U_{l}}\right)_{\rho_{l}}^{\mu_{l}}=S_{\mu_{l} \rho_{l}}^{U_{l}}$. In this case, Cardy's case, the matrices $n_{\nu_{l}}^{U_{l}}$ are just the fusion matrices. Details of these constructions can be found in [6, 31, 7] for instance.

Imposing trivial gluing conditions on all constituents of the reduced chiral algebra (2.2) enforces the condition $\mu_{l}=\nu_{l}=\Omega_{l}\left(\nu_{l}\right)$ for the symmetric part of $\mathcal{H}^{G}$ from which we may construct Ishibashi states $\left.\left|\mu_{n}, \cdots, \mu_{1}\right\rangle\right\rangle$ [20]. By comparison with the previous paragraph we are thus able to define boundary states

$$
\left.\left|\rho_{n}, \cdots, \rho_{1}\right\rangle=\sum \frac{\left(\psi^{U_{n}}\right)_{\rho_{n}}^{\mu_{n}}}{\sqrt{S_{0 \mu_{n}}^{U_{n}}}} \frac{\left(\psi^{U_{n-1}}\right)_{\rho_{n-1}}^{\mu_{n-1}}}{S_{0 \mu_{n-1}}^{U_{n-1}}} \cdots \frac{\left(\psi^{U_{2}}\right)_{\rho_{2}}^{\mu_{2}}}{S_{0 \mu_{2}}^{U_{2}}} \frac{\left(\psi^{U_{1}}\right)_{\rho_{1}}^{\mu_{1}}}{S_{0 \mu_{1}}^{U_{1}}}\left|\mu_{n}, \cdots, \mu_{1}\right\rangle\right\rangle .
$$

Up to this point we merely reviewed the algebraic method of constructing symmetry breaking boundary states [20]. We will now use this algebraic information to prove that these boundary states describe D-branes which are localized along the product of twisted conjugacy classes (2.4). The first argument is based on the coupling of closed strings to the brane. In the section 3.3 we will then analyze the spectrum of open strings.

It is convenient to think about the boundary state (3.8) as describing D-branes in the group manifold $U_{n} \times U_{n-1} \times U_{n-1} \times \cdots \times U_{1} \times U_{1}$ which is the basic constituent of $G^{\text {new }}$. This follows from the discussion in section 3.1 and the factorized structure of eq. (3.8). A similar proposal in the context of coset theories has been made in [27] (see also [34]). According to the general discussion of [9], the calculation of closed string couplings to the brane shows that the $U_{n}$-part of the boundary state (3.8) gives rise to a D-brane which is localized along the twisted conjugacy class in $\mathcal{C}_{f_{n}}^{U_{n}}\left(\Omega_{n}\right)$ in $U_{n}$. The element $f_{n}$ is obtained from exponentiation of the weight $\rho_{n}$ to the (symmetric) Cartan torus in $U_{n}$. This prescription also makes explicit the quantization conditions which have to be imposed on twisted conjugacy classes. The other parts in eq. (3.8) are more difficult to access as the coefficients possess an additional factor of $\left(S_{0 \mu_{l}}^{U_{l}}\right)^{1 / 2}$ in the denominator. One may, however, easily check that such coefficients appear when constructing D-branes in $U_{l} \times U_{l}$ if one uses the 
gluing automorphism $\Omega_{l}^{\prime}\left(u_{l}, u_{l}^{\prime}\right)=\left(u_{l}^{\prime}, \Omega_{l}\left(u_{l}\right)\right)$ [35]. This choice of automorphisms - in particular the exchange of the group factors - reflects the fact that the target space $G^{\text {new }}$ is defined as an asymmetric coset. Other choices of automorphisms would not lead to a geometry which is consistent with the identification (3.2). The coupling of closed strings to previously described branes shows that they are localized along the twisted conjugacy classes $\mathcal{C}_{f_{l}^{\prime}}^{U_{l} \times U_{l}}\left(\Omega_{l}^{\prime}\right)=\left\{\left(s_{l} f_{l}^{\prime} t_{l}^{-1}, t_{l} f_{l}^{\prime} \Omega_{l}\left(s_{l}^{-1}\right)\right)\right\}$ with $f_{l}^{\prime}$ determined by $\rho_{l}$ as like before [9]. By taking the product of the two entries, this set projects down to a twisted conjugacy class $\mathcal{C}_{f_{l}}^{U_{l}}\left(\Omega_{l}\right)$ in the group $U_{l}$ where $f_{l}=f_{l}^{\prime 2}$.

Let us summarize these results. We have argued that the D-branes which are described by the boundary state (3.8) are localized along a direct product of twisted conjugacy classes in the space $U_{n} \times U_{n-1} \times U_{n-1} \times \cdots \times U_{1} \times U_{1}$. Due to their symmetry properties they descend to the set $G^{\text {new }}$ which provides a valid target space reinterpretation. As all elements of $G^{\text {new }}$ may be brought to the form (3.3), we just recover the expression (2.4) for the geometry of twisted D-branes which entered the Lagrangian approach.

\subsection{The spectrum of open strings}

Our next task is to determine the spectrum of open strings which can end on a D-brane which is described by the boundary state (3.8). The result has to be compared with predictions which arise from geometric description afterwards. The calculation of closed string propagation between two boundary states of the form (3.8) by world sheet duality yields the open string Hilbert space

$$
\mathcal{H}_{\rho \rho}=\bigoplus\left(n_{\nu_{n}}^{U_{n}}\right)_{\rho_{n}}^{\rho_{n}}\left[\prod_{l=1}^{n-1} N_{\nu_{l} \sigma_{l}^{+}}^{\lambda_{l}}\left(n_{\lambda_{l}}^{U_{l}}\right)_{\rho_{l}}^{\rho_{l}}\right] \mathcal{H}_{\left(\nu_{n}, \sigma_{n-1}\right)}^{U_{n} / U_{n-1}} \otimes \cdots \otimes \mathcal{H}_{\left(\nu_{2}, \sigma_{1}\right)}^{U_{2} / U_{1}} \otimes \mathcal{H}_{\nu_{1}}^{U_{1}}
$$

where we used the abbreviation $\rho=\left(\rho_{1}, \cdots, \rho_{n}\right)$ to denote the boundary label. The NIM-reps $n^{U_{l}}$ have been defined in eq. (3.7) and the numbers $N$ are fusion coefficients of the affine Lie algebras $\hat{\mathfrak{u}}_{k_{l}}$. The calculation proceeds in the same way as those in [20].

In the geometric picture only the ground state structure of the Hilbert space (3.9) can be recovered. Let us denote by $\mathcal{H}_{\rho \rho}^{(0)}$ the set of all ground states which are present in eq. (3.9). Our aim is to find an explicit expression for this space which solely contains geometric information in the limit $k \rightarrow \infty$. The ground states of affine representations transform in a representation of the underlying simple Lie algebra which is usually denoted by the same symbol. In contrast, it is more difficult to give a geometrical meaning to the coset representations. All we can do is to determine the number of ground states they contain. The latter is given by the branching coefficients which describe the embedding of the associated horizontal subalgebras. These considerations provide a dictionary of how to extract geometrical information out of eq. (3.9). We simply have to replace affine

representations $\mathcal{H}_{\nu_{1}}^{U_{1}}$ by representation spaces $V_{\nu_{1}}^{U_{1}}$ of $\mathfrak{u}_{1}$ and branching spaces $\mathcal{H}_{\left(\nu_{l}, \sigma_{l-1}\right)}^{U_{l} / U_{l-1}}$ by branching coefficients of the embedding $\mathfrak{u}_{l-1} \hookrightarrow \mathfrak{u}_{l}$. When represented as a $H$ module we 
end up with the following expression,

$$
\mathcal{H}_{\rho \rho}^{(0)}=\bigoplus\left(n_{\nu_{n}}^{U_{n}}\right)_{\rho_{n}}^{\rho_{n}}\left[\prod_{l=1}^{n-1} N_{\nu_{l} \sigma_{l}^{+}}^{\lambda_{l}}\left(n_{\lambda_{l}}^{U_{l}}\right)_{\rho_{l}}^{\rho_{l}} b_{\nu_{l+1}} \sigma_{l}\right] V_{\nu_{1}}^{U_{1}}
$$

for the space of ground states. The geometric limit of a WZNW theory is obtained by sending the level $k$ of the affine Kac-Moody algebra $\hat{\mathfrak{g}}_{k}$ to infinity. This automatically forces the levels of the Kac-Moody subalgebras $\left(\hat{\mathfrak{u}}_{l}\right)_{k_{l}}$ also to tend to infinity. In this limit the fusion coefficients $N$ entering eq. (3.9) reduce to tensor product coefficients and also the NIM-reps $n^{U_{l}}$ have a natural geometrical meaning [14].

Let us now turn our attention to the evaluation of D-brane spectra in the geometric picture. As was shown in [13, 15] for ordinary conjugacy classes and then generalized to the twisted case in [14] there is always a noncommutative geometry associated to these objects. In some sense this reflects the geometric limit of the noncommutative algebra of open string vertex operators. For the ground states this algebra indeed becomes coordinate independent in the large volume limit $k \rightarrow \infty$ as their conformal dimensions tend to zero. For an arbitrary D-brane wrapped around the twisted conjugacy class $\mathcal{C}_{f_{l}}^{U_{l}}\left(\Omega_{l}\right)$ this noncommutative algebra admits an action of $U_{l}$ under which it decomposes into modules $V_{\nu_{l}}^{U_{l}}$ according to

$$
\mathcal{A}\left(\mathcal{C}_{f_{l}}^{U_{l}}\left(\Omega_{l}\right)\right)=\bigoplus\left(n_{\nu_{l}}\right)_{\rho_{l}}^{\rho_{l}} V_{\nu_{l}}^{U_{l}}
$$

The same expression would be obtained for $\operatorname{Inv}_{U_{l}} \mathcal{A}\left(\mathcal{C}_{f_{l}^{\prime}}^{U_{l} \times U_{l}}\left(\Omega_{l}^{\prime}\right)\right)$ where the invariance is defined with respect to the identification $\left(u_{l}^{\prime}, u_{l}\right) \sim\left(u_{l}^{\prime} \cdot t_{l}^{-1}, t_{l} \cdot u_{l}\right)$ for $t_{l} \in U_{l}$ (cf. eq. (3.2)) . For our further discussion it is not necessary to know the detailed form of the matrices $n^{U_{l}}$. It is only important to note that the numbers $n^{U_{l}}$ entering eq. (3.10) coincide with those in eq. (3.11) in the limit $k \rightarrow \infty$ [14].

The module structure of the algebra for a (direct) product of twisted conjugacy classes is given by the tensor product of the individual modules. We thus obtain the spectrum of open string ground states

$$
\mathcal{A}\left(\mathcal{D}\left\{U_{l}, \Omega_{l}, f_{l}\right\}\right)=\bigoplus_{\left\{\nu_{l}\right\}}\left[\prod_{l=1}^{n}\left(n_{\nu_{l}}\right)_{\rho_{l}}^{\rho_{l}}\right] V_{\nu_{n}}^{U_{n}} \otimes \cdots \otimes V_{\nu_{1}}^{U_{1}},
$$

interpreted as a module of the group $U_{n} \times \cdots \times U_{1}$. In our approach of section 2, the twisted conjugacy classes are not viewed as modules of the groups $U_{l}$ but as modules of the diagonally embedded $H=U_{1}$. This means that we should fully decompose the module (3.12) with respect to $H$ in order to read off the spectrum of ground states belonging to the D-brane described by $\mathcal{D}\left\{U_{l}, \Omega_{l}, f_{l}\right\}$. In this way we get a number of additional branching and tensor product coefficients.

It is now straightforward to check the equality of the expressions on the right hand side of eqs. (3.10) and (3.12) in the limit $k \rightarrow \infty$, both considered as $H$ modules. In 
other words we have just proven the relation $\mathcal{A}\left(\mathcal{D}\left\{U_{l}, \Omega_{l}, f_{l}\right\}\right) \cong \mathcal{H}_{\rho \rho}^{(0)}$ which expresses the agreement of the open string spectra obtained both from an algebraic and a geometric point of view, respectively. Let us emphasize that the geometric open string algebra $\mathcal{A}\left(\mathcal{D}\left\{U_{l}, \Omega_{l}, f_{l}\right\}\right)$ may not be identified with the algebra of functions on the D-brane world volume $\mathcal{D}\left\{U_{l}, \Omega_{l}, f_{l}\right\} \subset G$. Again this may be interpreted as an effect in favour of working with the new target space $G^{\text {new }}$. When considered as an object in $G$, the points in the D-brane world volume can be covered more than once. Obviously it is not possible to describe the new degrees of freedom which are associated to such multiple wrappings and superpositions of D-branes by the usual algebra of functions on the world volume $\mathcal{D}\left\{U_{l}, \Omega_{l}, f_{l}\right\} \subset G$. Similar observations have been discussed in [17].

\section{Applications}

In this section we will apply our general results to advance the classification of D-branes in the target space $G=S L(2, \mathbb{R}) \times S U(2)$. When lifted to the covering space $A d S_{3}$ of $S L(2, \mathbb{R})$ these provide us with non-factorizing and symmetry breaking D-branes in the string backgrounds $A d S_{3} \times S^{3} \times T^{4}$ and $A d S_{3} \times S^{3} \times S^{3} \times S^{1}$. The CFT description of WZNW models for noncompact groups is very intricate as their spectrum is continuous. In particular their analysis is not covered by [20]. We have therefore nothing new to say about the CFT side of constructing D-branes in these backgrounds. Nevertheless we conjecture that at least qualitatively our geometric analysis reflects the correct picture. Parts of our observations for factorizing D-branes in $S L(2, \mathbb{R}) \times S U(2)$ are immediate consequences

of earlier results which have been obtained by different methods [22, 36, 23, 17, 24, 21]. For the sake of completeness and in order to illustrate the power of our new geometric description we also review these cases. In particular we will show that our approach confirms the T-duality prediction for the geometry of symmetry breaking D-branes in $S L(2, \mathbb{R})$ and $S U(2)[17,21]$.

According to the general scheme we have first to find out all inequivalent embeddings of continuous subgroups into each of the constituents $S L(2, \mathbb{R})$ and $S U(2)$. In addition

we have to classify all the automorphisms of these subgroups. Common subgroups of $S L(2, \mathbb{R})$ and $S U(2)$ can then be used to construct non-factorizing symmetry breaking D-branes.

\subsection{Preliminaries}

The continuous subgroups of the product $S L(2, \mathbb{R}) \times S U(2)$ are easily classified. There are essentially two choices of embedding chains (2.1) which may be used for our construction. The first one is given by the maximal embedding

$$
H_{1} \times H_{2} \hookrightarrow S L(2, \mathbb{R}) \times S U(2)
$$


where $H_{1}$ and $H_{2}$ equal one of the groups $U(1)$ or $\mathbb{R}$. Without loss of generality we assume that $H_{1}$ is embedded into $S L(2, \mathbb{R})$ and $H_{2}$ is embedded into $S U(2)$. In this case the embedding map is given by $\epsilon^{H_{1}, S L} \times \epsilon^{H_{2}, S U}$. To save space we used the abbreviations $S L$ and $S U$ for $S L(2, \mathbb{R})$ and $S U(2)$, respectively. Before we dive into the discussion of these maps, let us first write down the second choice for a chain of embeddings. It is specified by

$$
H \hookrightarrow H \times H \hookrightarrow S L(2, \mathbb{R}) \times S U(2)
$$

Again, the symbol $H$ denotes a group of type $U(1)$ or $\mathbb{R}$. The embedding map can be written as $\left[\epsilon^{H, S L} \times \epsilon^{H, S U}\right] \circ \epsilon^{H, H \times H}$. The decomposition (2.2) of chiral algebras which arises from (4.2) contains cosets of the form $\mathcal{A}(H / H)$ if $H$ is completely embedded in one of the factors $H \times H$. As these are nasty to deal with on the algebraic level, we assume all possible embeddings in relation (4.2) to have the diagonal form $\epsilon^{H, H \times H}(h)=(h, h)$. The remaining freedom is then contained in the embedding maps $\epsilon^{H, S L} \times \epsilon^{H, S U}$ from $H \times H$ to $S L(2, \mathbb{R}) \times S U(2)$.

Our next task is to classify all embeddings and automorphisms of groups and subgroups belonging to the relations (4.1) and (4.2). Readers familiar with these issues may directly jump to section 4.2. The embeddings for the subgroup $S O(2) \cong U(1)$ are given by

$$
\epsilon_{n}^{U, S L}\left(e^{i \phi}\right)=\epsilon_{n}^{U, S U}\left(e^{i \phi}\right)=\left(\begin{array}{cc}
\cos n \phi & \sin n \phi \\
-\sin n \phi & \cos n \phi
\end{array}\right) \quad \text { for } n \in \mathbb{Z} \backslash\{0\}
$$

In addition both groups admit an embedding of $\mathbb{R}$. For $S L(2, \mathbb{R})$ we have two inequivalent choices one of which utilizes the covering of $U(1)$ by $\mathbb{R}$. The latter also allows an embedding of $\mathbb{R}$ into $S U(2)$. The explicit maps read

$$
\epsilon_{\alpha}^{\mathbb{R}, S L}(\lambda)=\left(\begin{array}{cc}
e^{\alpha \lambda} & 0 \\
0 & e^{-\alpha \lambda}
\end{array}\right) \quad \text { and } \quad \tilde{\epsilon}_{\beta}^{\mathbb{R}, S L}(\lambda)=\tilde{\epsilon}_{\beta}^{\mathbb{R}, S U}(\lambda)=\left(\begin{array}{cc}
\cos \beta \lambda & \sin \beta \lambda \\
-\sin \beta \lambda & \cos \beta \lambda
\end{array}\right) .
$$

Obviously, we have to demand that $\alpha$ and $\beta$ are non-vanishing.

The automorphisms are also easily classified. The group $S U(2)$ only admits inner automorphisms. According to the remarks at the end of section 2, we may assume them to act trivially without any restrictions. In contrast, there exist outer automorphisms of the group $S L(2, \mathbb{R})$ which are based on the representative

$$
\Omega_{0}^{S L}\left(\begin{array}{ll}
a & b \\
c & d
\end{array}\right)=\left(\begin{array}{ll}
d & c \\
b & a
\end{array}\right)=\left(\begin{array}{ll}
0 & 1 \\
1 & 0
\end{array}\right)\left(\begin{array}{ll}
a & b \\
c & d
\end{array}\right)\left(\begin{array}{ll}
0 & 1 \\
1 & 0
\end{array}\right)
$$

Note that this automorphism allows a representation as conjugation with the matrix $M=\left(\begin{array}{ll}0 & 1 \\ 1 & 0\end{array}\right)$ which is not an element of $S L(2, \mathbb{R})$. For the subgroups $U(1)$ and $\mathbb{R}$ we obtain

$$
\Omega_{ \pm}^{U}\left(e^{i \phi}\right)=e^{ \pm i \phi} \quad \text { and } \quad \Omega_{ \pm}^{\mathbb{R}}(\lambda)= \pm \lambda
$$


In fact, we could choose for $\mathbb{R}$ the multiplication with an arbitrary non-zero real number. In our description we implicitly demanded, however, that the automorphisms should be consistent with the scalar product in the Lie algebra, i.e. they should not change the embedding index.2 This condition restricts our possibilities to the multiplication by \pm 1 . Finally, we have to discuss the automorphisms of $H \times H$. In this paper we restrict ourselves to automorphisms of the form $\Omega^{H \times H}=\Omega \circ\left[\Omega_{1}^{H} \times \Omega_{2}^{H}\right]$, where $\Omega: H \times H \rightarrow H \times H$ denotes a possible exchange of the two group factors and $\Omega_{1}^{H}, \Omega_{2}^{H}$ are two arbitrary automorphisms of $H$. If $H$ would have been semi-simple our choice of $\Omega^{H \times H}$ would have exhausted all possibilities. For our abelian groups $U(1)$ and $\mathbb{R}$, there are more general choices but they are generically plagued with some unwanted features.

We are now almost prepared to address the question of D-brane geometry in $S L(2, \mathbb{R}) \times$ $S U(2)$. All we still need is a better understanding of certain twisted conjugacy classes. Let $H$ be one of the subgroups $U(1)$ or $\mathbb{R}$. As both groups are abelian one immediatley obtains

$$
\mathcal{C}_{f}^{H}\left(\Omega_{\eta}^{H}\right)=\left\{\begin{array}{cl}
\{f\} & , \eta=+ \\
H & , \eta=-
\end{array}\right.
$$

The element $f$ has to satisfy the symmetry property $\Omega_{\eta}^{H}(f)=f$. It is slightly more complicated to find an expression for the twisted conjugacy classes $\mathcal{C}^{H \times H}\left(\Omega^{H \times H}\right)$. Considering first the case with $\Omega=$ id we easily obtain

$$
\left.\mathcal{C}_{\left(f_{1}, f_{2}\right)}^{H \times H}\left(\Omega^{H \times H}\right)\right|_{\Omega=\mathrm{id}}=\left\{\begin{array}{cl}
\left\{f_{1}\right\} \times\left\{f_{2}\right\} & , \Omega_{1}^{H}=\mathrm{id}, \Omega_{2}^{H}=\mathrm{id} \\
\left\{f_{1}\right\} \times H & , \Omega_{1}^{H}=\mathrm{id}, \Omega_{2}^{H} \neq \mathrm{id} \\
H \times\left\{f_{2}\right\} & , \Omega_{1}^{H} \neq \mathrm{id}, \Omega_{2}^{H}=\mathrm{id} \\
H \times H & , \Omega_{1}^{H} \neq \mathrm{id}, \Omega_{2}^{H} \neq \mathrm{id}
\end{array}\right.
$$

In this case the element $\left(f_{1}, f_{2}\right)$ has to satisfy $\Omega_{i}^{H}\left(f_{i}\right)=f_{i}$. With non-trivial twist, i.e. with $\Omega \neq$ id, we have the restrictions $f_{1}=\Omega_{2}^{H}\left(f_{2}\right)$ and $f_{2}=\Omega_{1}^{H}\left(f_{1}\right)$. It thus suffices to work with one label $f=f_{1}$ which satisfies $\Omega_{2}^{H} \circ \Omega_{1}^{H}(f)=f$. A straightforward analysis yields

$$
\left.\mathcal{C}_{f}^{H \times H}\left(\Omega^{H \times H}\right)\right|_{\Omega \neq \mathrm{id}}=\left\{\begin{array}{cl}
H \times H & , \Omega_{1}^{H} \neq\left(\Omega_{2}^{H}\right)^{-1} \text { and } \Omega \neq \mathrm{id} \\
\left\{\left(s f, \Omega_{1}^{H}\left(f s^{-1}\right)\right) \mid s \in H\right\} & , \Omega_{1}^{H}=\left(\Omega_{2}^{H}\right)^{-1} \text { and } \Omega \neq \mathrm{id} .
\end{array}\right.
$$

This concludes our presentation of the necessary tools for the determination of symmetry breaking D-branes in $S L(2, \mathbb{R}) \times S U(2)$.

\footnotetext{
${ }^{2}$ This is automatically satisfied for all semi-simple groups where outer automorphisms arise from Dynkin diagram symmetries.

${ }^{3}$ The scalar product in the corresponding Lie algebra would not be invariant.
} 


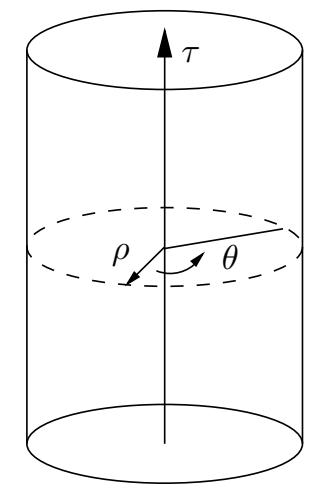

Figure 1: Parametrization of $S L(2, \mathbb{R})$.

\subsection{Symmetry breaking D-branes in $S L(2, \mathbb{R})$ and $S U(2)$ - a re- view}

This subsection will be used to introduce some notation which is necessary to describe the geometry of $S L(2, \mathbb{R})$ and $S U(2)$. In addition we will find that our approach is in agreement with recent results for symmetry breaking D-branes in these groups which have been obtained using T-duality [17, 24, 21].

\subsubsection{Symmetry breaking D-branes in $S L(2, \mathbb{R})$}

The group $S L(2, \mathbb{R})$ may be described as a subspace of four dimensional flat space. In this parametrization the connection to the matrix form is given by

$$
\left(\begin{array}{ll}
X_{0}+X_{3} & X_{1}+X_{2} \\
X_{1}-X_{2} & X_{0}-X_{3}
\end{array}\right) \quad \text { subject to } \quad X_{0}^{2}-X_{1}^{2}+X_{2}^{2}-X_{3}^{2}=1 .
$$

It is convenient to introduce cylindrical coordinates $r, \theta$ and a periodic time $\tau$. These take values in the domains $r \in[0, \infty[$ and $\theta, \tau \in[0,2 \pi[$. The precise relation to the previous parametrization is given by

$$
X_{0}+i X_{2}=e^{i \tau} \cosh r \quad \text { and } \quad X_{3}+i X_{1}=e^{i \theta} \sinh r
$$

In the cylindrical coordinates the manifold $S L(2, \mathbb{R})$ may be depicted as in figure 1 with top and bottom of the cylinder identified. The covering space $A d S_{3}$ is obtained by resolving the periodicity of time, i.e. by extending its range to $\tau \in \mathbb{R}$. The region $r \rightarrow \infty$ describes the boundary of $\mathrm{AdS}_{3}$.

Maximally symmetric D-branes belong to twisted conjugacy classes of $S L(2, \mathbb{R})$. For ordinary conjugacy classes all elements are mapped to the same number by taking the trace. Fixing $X_{0}$ to some value $C \in \mathbb{R}$ while putting no constraints on the other coordinates therefore gives a first rough classification. The resulting submanifold may be disconnected showing that only demanding $X_{0}=C$ does not lead to a complete solution of the classification problem. It is nevertheless useful to work with this description. The 

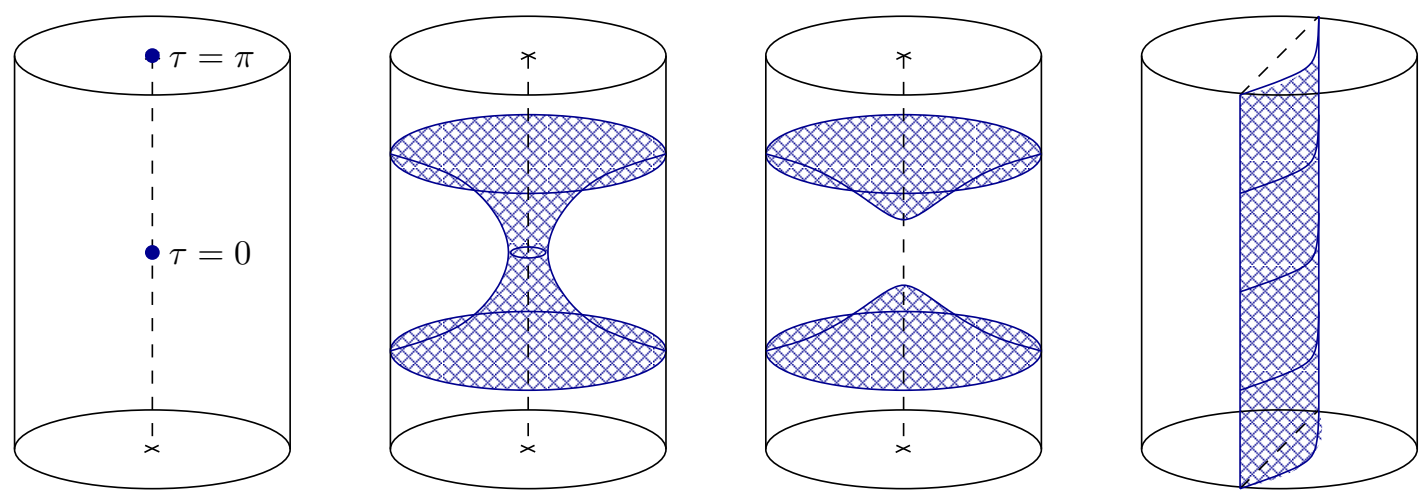

Figure 2: Representatives of maximally symmetric D-branes in $S L(2, \mathbb{R})$. From left to right we have the following types: point-like, $d S_{2}, H_{2}$ and $A d S_{2}$ branes.

equation $X_{0}=\cos \tau \cosh r=C$ admits very different types of solutions depending on whether $|C|<1$ or $|C|>1$. For $|C|>1$ one recovers $d S_{2}$ branes while for $|C|<1$ branes are obtained which are localized on hyperbolic planes $H_{2}$. In the limit $|C| \rightarrow 1$ they degenerate to two instantonic point-like D-branes at $\tau=0, \pi$ which are associated to the center of $S L(2, \mathbb{R})$ and others sitting on the light cone. Representatives of this zoo of conjugacy classes are visualized in figure 2. These results have already been found in [22, 23]. It was argued in [23] that all these D-branes are unphysical. The $H_{2}$ and the point-like branes are instantonic objects while the $d S_{2}$ branes are spoiled by a supercritical electrical field.

Twisted conjugacy classes coming from the automorphism (4.4) are classified by the relation $\operatorname{tr}(M g)=2 X_{2}=2 C$. According to eq. (4.9) this translates into $C=\sin \theta \sinh r$. In this situation there is no need to distinguish different cases. All these twisted conjugacy classes describe $A d S_{2}$ branes which are invariant under time translations and extend to the boundary of $A d S_{3}$ at $\theta=0, \pi[23]$. They are illustrated in the right-most picture of figure 2 .

Let us now turn to the description of symmetry breaking D-branes. According to the expression (2.4) we may multiply the twisted conjugacy classes of $S L(2, \mathbb{R})$ by a twisted conjugacy class $\mathcal{C}_{f}^{H}\left(\Omega^{H}\right)$ of $H=U(1)$ or $H=\mathbb{R}$. For $\Omega^{H}=$ id the latter are point-like. This induces a shift of the original D-brane. The situation is more interesting if $\Omega^{H} \neq \mathrm{id}$. In this case the twisted conjugacy class reduces to $H$ itself and one has to consider the superposition of all shifted images.

This analysis is particularly simple for $H=U(1)$. In this case the multiplication of an element $(r, \theta, \tau)$ of $S L(2, \mathbb{R})$ with an element $e^{i \lambda}$ of $U(1)$ just induces the simultaneous rotation $(\theta, \tau) \mapsto(\theta \pm n \lambda, \tau \pm n \lambda)$ of angle and time coordinate. The sign and the wrapping number $n$ are fixed by the choice of twist $\Omega^{S L}$ and embedding $\epsilon^{U, S L}$. As the twisted conjugacy class is given by the whole $U(1)$, one may immediately evaluate the geometry of the resulting D-branes. By rotation of the $d S_{2}$ and the $A d S_{2}$ branes one obtains D- 

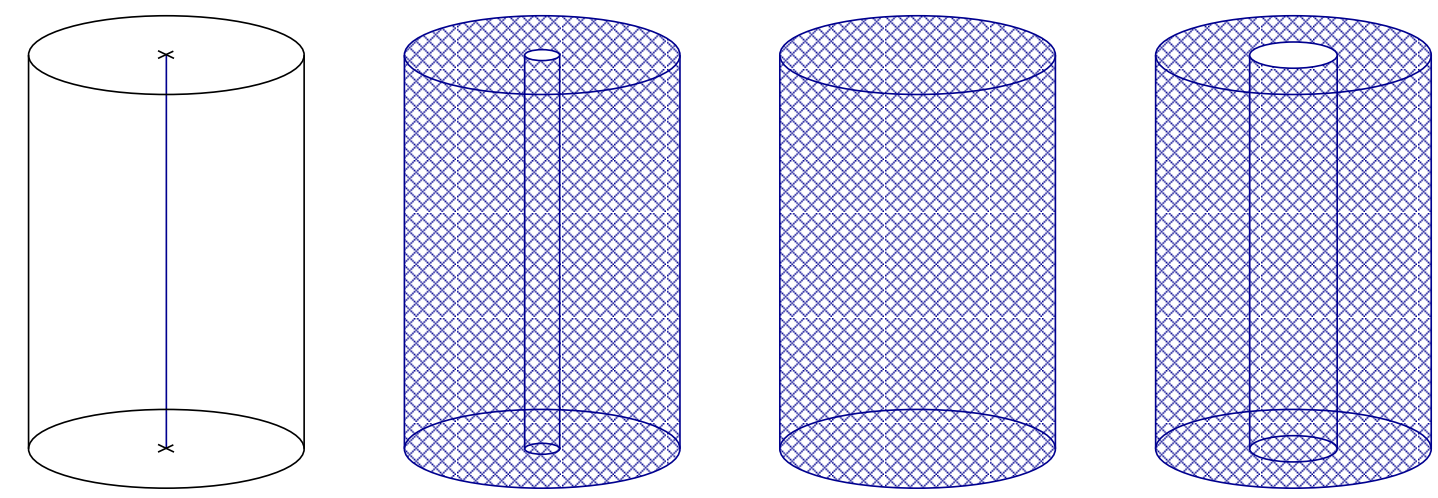

Figure 3: Certain classes of symmetry breaking D-branes on $S L(2, \mathbb{R})$. They are obtained from those in figure 2 by a simultaneous $(\theta, \tau)$-rotation.

branes which fill all space outside a cylinder of radius $r_{0}=\operatorname{arcosh}|C|$ or $r_{0}=\operatorname{arsinh}|C|$, respectively. For degenerate cases they provide space-filling branes similar to those arising from the rotation of $\mathrm{H}_{2}$ branes. If one rotates the 0-branes on the other hand these sweep out the axis $r=0$. To get some impression of these geometries we visualized all four of them in figure 2. Let us emphasize that the generic point in the world volume of rotated $d S_{2}$ and $A d S_{2}$ branes is covered twice.

For $H=\mathbb{R}$ we have to distinguish two embeddings (4.3). The usage of $\tilde{\epsilon}_{\beta}^{\mathbb{R}, S L}$ gives essentially the same result as for $U(1)$. For $\epsilon_{\beta}^{\mathbb{R}, S L}$, in contrast, the discussion becomes quite involved as the shift acts in a very intricate way - at least in our coordinates $(r, \theta, \tau)$. To get an idea of what is going, on let us consider the case where the conjugacy class of $S L(2, \mathbb{R})$ reduces to a point. The D-brane is then parametrized by matrices of the form $\operatorname{diag}\left( \pm e^{\lambda}, \pm e^{-\lambda}\right)$ with $\lambda \in \mathbb{R}$. It turns out that these are instantonic D1-branes localized at times $\tau=0, \pi$, respectively, and running all the way from $r=0$ to $r=\infty$ in the directions $\theta=0, \pi$. They do not seem to make sense physically and we will not discuss them in more detail. Notice that most of the symmetry breaking D-branes which have been described in this section already appeared in [17, 24, 21].

\subsubsection{Symmetry breaking D-branes in $S U(2)$}

The group manifold $S U(2)$ may be realized as a subset of $\mathbb{C}^{2}$. In this parametrization the elements are described by a matrix $\left(\begin{array}{cc}z_{1} & z_{2} \\ -\bar{z}_{2} & \bar{z}_{1}\end{array}\right)$ subject to the condition $\left|z_{1}\right|^{2}+\left|z_{2}\right|^{2}=1$. Maximally symmetric D-branes are localized along quantized conjugacy classes [8, 9]. For a WZNW theory at level $k$ we have $k+1$ spheres $S^{2}$ which sit at the special values $\operatorname{Re}\left(z_{1}\right)=\cos \frac{\pi \mu}{k}$ with $\mu=0, \cdots, k$. For $\mu=0, k$ they degenerate to points. An illustration of these facts is given on the left hand side of figure 4 .

To describe symmetry breaking D-branes we have to multiply these conjugacy classes by twisted conjugacy classes of $U(1)$ or $\mathbb{R}$. Choosing a trivial automorphism amounts to a shift like before. When considering a non-trivial automorphism we have to take the union 

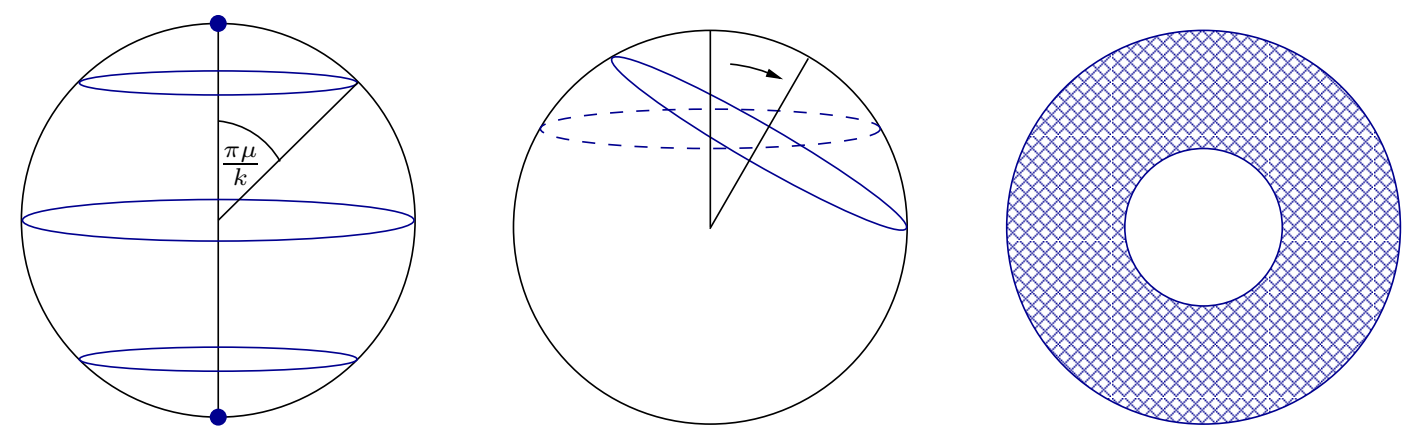

Figure 4: Maximally symmetric and symmetry breaking D-branes on $S U(2)$. The latter arise from the rotation indicated in the central picture. They generically cover a 3dimensional subset of $S^{3}$ but leave open a "window" of a certain size.

of all these shifted images. We will not write down the explicit expressions but only refer to the illustration on the right hand side of figure 6 . The symmetry breaking D-branes are either 1- or 3-dimensional. While the first ones are circular, the latter cover most of the group but generically leave some parts uncovered. Let us emphasize that we also find a space-filling brane by considering the conjugacy classes of $S U(2)$ with $\mu=k / 2$ for even values of $k$. It is remarkable to note that a generic point of all these D-branes is covered twice. This observation is related to the fact that the space-filling brane can be further resolved into two single branes. These results are not new but have already been obtained in [17, 21] with different methods.

\subsection{On the hierarchy of D-branes in $S L(2, \mathbb{R}) \times S U(2)$}

When constructing D-branes in the product geometry $S L(2, \mathbb{R}) \times S U(2)$, it is convenient to distinguish three cases which belong to qualitatively different classes of automorphisms for the subgroups appearing in the two embedding chains (4.1) and (4.2). The discussion of this classification will be the subject of the following three subsections.

\subsubsection{Factorizing D-branes}

Factorizing D-branes arise from the embedding chain (4.1). For $H=H_{1}=H_{2}$ we further have to assume that the automorphism of $H \times H$ involved in the construction of the Dbranes does not contain the exchange of the group factors. Under these circumstances the decomposition (2.2) reduces to a separate decomposition of $\mathcal{A}(S L(2, \mathbb{R}))$ and $\mathcal{A}(S U(2))$ in both holomorphic and antiholomorphic degrees of freedom.

Let us discuss the geometry of these D-branes now. According to the general expression (2.4) they are localized along the product

$$
\left[\mathcal{C}_{f_{1}}^{S L}\left(\Omega^{S L}\right) \cdot \Omega^{S L} \circ \epsilon^{H_{1}, S L}\left(\mathcal{C}_{f_{2}}^{H_{1}}\left(\Omega^{H_{1}}\right)\right)\right] \times\left[\mathcal{C}_{f_{3}}^{S U}(\mathrm{id}) \cdot \epsilon^{H_{2}, S U}\left(\mathcal{C}_{f_{4}}^{H_{2}}\left(\Omega^{H_{2}}\right)\right)\right] .
$$


This factorized geometry is completely under control using the dictionary which has been provided in section 4.2. The dimensions of these D-branes range from 0 to 6 , the shape from point-like to space-filling. We will not bother to discuss these D-branes any further but focus our attention on the description of non-factorizing D-branes.

\subsubsection{Non-factorizing D-branes from diagonal embedding}

The first type of non-factorizing D-branes is obtained by using the embedding chain (4.2) and choosing an automorphism $\Omega^{H \times H}$ which does not involve an exchange of the two factors. In other words we demand $\Omega=$ id. The geometry associated to this kind of symmetry breaking D-brane is described by the product

$$
\left[\mathcal{C}_{f_{1}}^{S L}\left(\Omega^{S L}\right) \times \mathcal{C}_{f_{2}}^{S U}(\mathrm{id})\right] \cdot \epsilon_{1}\left(\mathcal{C}_{\left(f_{3}, f_{4}\right)}^{H \times{ }_{1}}\left(\Omega^{H \times H}\right)\right) \cdot \epsilon_{2}\left(\mathcal{C}_{f_{5}}^{H}\left(\Omega^{H}\right)\right)
$$

where the embeddings have been abbreviated by $\epsilon_{1}=\left(\Omega^{S L} \circ \epsilon^{H, S L}\right) \times \epsilon^{H, S U}$ and $\epsilon_{2}=$ $\left[\Omega^{S L} \circ \epsilon^{H, S L} \times \epsilon^{H, S U}\right] \circ \Omega^{H \times H} \circ \epsilon^{H, H \times H}$. Let us start our discussion with a given product of conjugacy classes of $S L(2, \mathbb{R})$ and $S U(2)$. As can be seen from eq. (4.6) the effect of the multiplication with $\mathcal{C}_{\left(f_{3}, f_{4}\right)}^{H \times H}\left(\Omega^{H \times H}\right)$ is a combination of a factorized smearing as described in section 4.2 and a translation. The effect of the multiplication with $\mathcal{C}_{f_{5}}^{H}\left(\Omega^{H}\right)$ is more interesting as it can provide the reason for non-factorizability. If this conjugacy class is 0-dimensional it shifts the whole D-brane by a constant amount leaving factorizability unaffected. On the other hand it may reduce to $H$ itself. Under these circumstances one obtains a continuous superposition of shifted D-branes. Due to the diagonal embedding of $H$ into $H \times H$, the shift acts on both factors $S L(2, \mathbb{R})$ and $S U(2)$ simultaneously. This feature is responsible for non-factorizability.

The discussion of the geometry of these D-branes becomes rather involved in the general case. We prefer to illustrate our considerations in two simple examples. Assume first that $H=\mathbb{R}, \epsilon^{H, S L}=\tilde{\epsilon}_{\alpha}^{\mathbb{R}, S L}$ and that we set $f_{1}=f_{2}=f_{3}=f_{4}=e, \Omega^{S L}=$ id and $\Omega_{1}^{H}=\Omega_{2}^{H}=$ id. This implies that the twisted conjugacy classes of $S L(2, \mathbb{R}), S U(2)$ and $H \times H$ entering (4.10) reduce to unit elements. In order to obtain a non-trivial result we choose $\Omega^{H} \neq$ id such that the remaining twisted conjugacy class in eq. (4.10) is given by $H$. After its embedding into $S L(2, \mathbb{R}) \times S U(2)$ one recovers the curve

$$
\left(\left(\begin{array}{cc}
\cos \alpha \lambda & \sin \alpha \lambda \\
-\sin \alpha \lambda & \cos \alpha \lambda
\end{array}\right),\left(\begin{array}{cc}
\cos \beta \lambda & \sin \beta \lambda \\
-\sin \beta \lambda & \cos \beta \lambda
\end{array}\right)\right) \quad \text { with } \lambda \in \mathbb{R} .
$$

The numbers $\alpha, \beta$ specifiy the individual embeddings of $\mathbb{R}$ into the group factors. A closer comparison of this expression with eqs. (4.8,4.9) shows that $\alpha \lambda$ may be identified with time $\tau$. This configuration thus describes a number of D-particles each having a circular trajectory in the group factor $S U(2)$ while sitting on the axis $r=0$ of $S L(2, \mathbb{R})$. The number of D-particles is determined by the relative values of $\alpha$ and $\beta$. If the ratio is irrational one obtains an infinite number of particles which form a dense set in $S U(2)$ at 
each instance of time. The appearance of multiple D-particles is due to the periodicity of time in $S L(2, \mathbb{R})$. This artefact disappears on the covering space $A d S_{3}$ which has a non-compact time-coordinate. Obviously, it is straightforward to generalize the previous idea to 2-spheres which are rotating in the $S U(2)$ factor in the evolution of time.

In our second example we choose $\Omega^{S L}=\Omega_{0}^{S L}$, but still fix $H=\mathbb{R}, \epsilon^{H, S L}=\tilde{\epsilon}_{\alpha}^{\mathbb{R}, S L}$, $f_{3}=f_{4}=e, \Omega_{1}^{H}=\Omega_{2}^{H}=\mathrm{id}$ and $\Omega^{H} \neq \mathrm{id}$. The set (4.10) is obtained from the product $A d S_{2} \times S^{2}$ by performing simultaneous shifts in both factors. If we focus only on the $A d S_{2}$-part for a moment we already know the resulting geometry from section 4.2.1. It is given by all points $(r, \theta, \tau)$ which satisfy $r \geq r_{0}=\operatorname{arsinh}|C|$ for some constant $C$. These points are generically not located on the twisted conjugacy class of $S L(2, \mathbb{R})$ we have started from. We thus have to decompose them into an element $\left(r, \theta^{\prime}, \tau^{\prime}\right)$ and a shift $\lambda$ such that $(\theta, \tau)=\left(\theta^{\prime}+\lambda, \tau^{\prime}+\lambda\right)$ and $\sin \theta^{\prime} \sinh r=C$, i.e. such that $\left(r, \theta^{\prime}, \tau^{\prime}\right)$ is an element of the twisted conjugacy class. With every solution $\theta_{1}^{\prime}$ we have another one $\theta_{2}^{\prime}=\pi-\theta_{1}^{\prime}$. In the exceptional case $r=r_{0}$ we have only one solution $\theta_{1}^{\prime}=\theta_{2}^{\prime}=\pi / 2$. For $r=r_{0}=0$ the angle can be chosen arbitrary. For simplicity we shall assume $r_{0}>0$ in what follows.

The two shifts $\lambda_{1 / 2}$ associated to the angles $\theta_{1 / 2}^{\prime}$ have to come from the embedding $\tilde{\epsilon}_{\alpha}^{\mathbb{R}, S L}$ of $\xi \in \mathbb{R}$ in $S L(2, \mathbb{R})$. As $\alpha \xi_{1 / 2}$ is only defined modulo $2 \pi$ there are several choices $\xi_{1 / 2}^{(l)}=\left(\lambda_{1 / 2}+2 \pi \alpha l\right) / \alpha$ of elements in $\mathbb{R}$ which may be used to recover these shifts. These elements have to be used to implement the shift on the $S U(2)$-part. Using the embedding $\tilde{\epsilon}_{\beta}^{\mathbb{R}, S U}$ as before, these shifts are determined by the angles $\beta\left(\lambda_{1 / 2}+2 \pi \alpha l\right) / \alpha$. For $\alpha=\beta=1$ we arrive at the following picture. The D-brane in $S L(2, \mathbb{R}) \times S U(2)$ is parametrized by points $(r, \theta, \tau)$ in $S U(2, \mathbb{R})$ with $r \geq r_{0}$. Over each of these points one has two spheres $S^{2}$ which are generated out of the conjugacy class of $S U(2)$ by the action of the shifts $\lambda_{1 / 2}(r)$. In the limiting regimes $r \rightarrow r_{0}$ and $r \rightarrow \infty$ the two-spheres move closer and closer. For more general choices of $\alpha$ and $\beta$ the number of two-spheres over each point in $S L(2, \mathbb{R})$ may be larger.

\subsubsection{Non-factorizing D-branes from group interchanging twists}

One can also consider the embedding chain (4.2) having a twist $\Omega \neq$ id of the $H \times H$ subgroup which interchanges the two factors. From the geometric point of view there are no difficulties in doing so. But at this point we have to remember that geometric automorphisms should be related to gluing automorphisms of currents on the level of chiral algebras. These chiral algebras are generated by affine Kac-Moody algebras and thus have the additional notion of a level. Consequently, the chiral algebras $\mathcal{A}(H)$ may differ depending on whether they come from an embedding of $H$ in $S L(2, \mathbb{R})$ or $S U(2)$. The geometric twist of $H \times H$ can only be lifted to an automorphism of $\mathcal{A}_{S L}(H) \oplus \mathcal{A}_{S U}(H)$ if these two algebras agree. This enforces some constraints on the relative size - the levels - of $S L(2, \mathbb{R})$ and $S U(2)$ and the embeddings one uses.

After these remarks we can proceed as in the previous section. The geometry which 
belongs to our present choice of embedding chain may be read off from the product

$$
\left[\mathcal{C}_{f_{1}}^{S L}\left(\Omega^{S L}\right) \times \mathcal{C}_{f_{2}}^{S U}(\mathrm{id})\right] \cdot \epsilon_{1}\left(\mathcal{C}_{f_{3}}^{H \times H}\left(\Omega^{H \times H}\right)\right) \cdot \epsilon_{2}\left(\mathcal{C}_{f_{4}}^{H}\left(\Omega^{H}\right)\right)
$$

where we used the abbreviations $\epsilon_{1}=\left(\Omega^{S L} \circ \epsilon^{H, S L}\right) \times \epsilon^{H, S U}$ and $\epsilon_{2}=\left[\Omega^{S L} \circ \epsilon^{H, S L} \times\right.$ $\left.\epsilon^{H, S U}\right] \circ \Omega^{H \times H} \circ \epsilon^{H, H \times H}$. The discussion of the conjugacy class $\mathcal{C}_{f_{4}}^{H}\left(\Omega^{H}\right)$ gives no new insights compared to the previous section. Despite of this fact there is still a significant qualitative difference, as we are now allowed to work with the expressions (4.7) for the conjugacy classes $\mathcal{C}_{f_{3}}^{H \times H}\left(\Omega^{H \times H}\right)$. While the first possibility implies the usual factorized smearing, the second induces a superposition of simultaneous shifts in the two group factors similar to those arising possibly from $\mathcal{C}_{f_{4}}^{H}\left(\Omega^{H}\right)$. It is an interesting question to see whether the joint action of two independent simultaneous shifts will lead to new features.

To illustrate these considerations we choose a setup where $H=\mathbb{R}, \epsilon^{H, S L}=\tilde{\epsilon}_{\alpha}^{\mathbb{R}, S L}$, $f_{1}=f_{2}=e, \Omega^{S L}=\mathrm{id}$ and $\Omega_{1}^{H}=\Omega_{2}^{H}=\Omega_{\eta}^{H}$ for $\eta= \pm 1$ as well as $\Omega^{H} \neq \mathrm{id}$. The product of the embedding of the two twisted conjugacy classes (4.11) is parametrized by two real numbers $\lambda, \lambda^{\prime}$ and reads

$$
\left(\left(\begin{array}{cc}
\cos \psi & \sin \psi \\
-\sin \psi & \cos \psi
\end{array}\right),\left(\begin{array}{cc}
\cos \psi^{\prime} & \sin \psi^{\prime} \\
-\sin \psi^{\prime} & \cos \psi^{\prime}
\end{array}\right)\right)
$$

with $\psi=\alpha\left(\eta \lambda+\lambda^{\prime}+f_{3}\right)$ and $\psi^{\prime}=\beta \eta\left(\lambda-\lambda^{\prime}+f_{3}\right)$. We recognize that the joint action of simultaneous shifts for $\eta=1$ leads to a factorized structure again as both $\psi$ and $\psi^{\prime}$ are independent. For $\eta=-1$ they become dependent and one recovers a shifted version of the already familiar non-factorizing D-brane instead.

\section{Conclusions}

In the present work we constructed the boundary WZNW functional for D-branes which are localized along products of generalized twisted conjugacy classes of subgroups $U_{l}$ of $G$ which are organized in an embedding chain of the form (2.1). The action functional was shown to be invariant under the action of a continuous subgroup. The D-branes have been identified as geometric counterparts of symmetry breaking boundary states constructed algebraically in [20]. Our results yield a huge hierarchy of D-branes in group manifolds which are under complete control both from an algebraic and a geometric point of view.

We focused on the example of $S L(2, \mathbb{R}) \times S U(2)$ to expedite the classification of Dbranes in this background. Although the groups $S L(2, \mathbb{R})$ and $S U(2)$ only possess a small number of subgroups which in addition are abelian, we perceived a glimpse of the possibilities which open up for more complicated backgrounds. In particular we are now able to work out the geometry of D-branes in product groups which do not necessarily factorize [20] and which do not originate from a twist of two group factors with equal 
size [36, 35]. The same ideas allow us to investigate defect lines in $1+1$-dimensional quantum field theories [20]. Such defects are for example induced on the holographic dual of $A d S_{3}$ by D-branes in its interior which extend to the boundary [37, 38, 39, 40]. It would be interesting to see whether our observations for D-branes in the non-compact group $S L(2, \mathbb{R}) \times S U(2)$ can be confirmed by an algebraic construction of boundary states.

Our algebraic analysis implied the necessity of target space reinterpretation in order to resolve certain ambiguities in the geometric description of symmetry breaking D-branes. In this way we revealed a striking connection between string theory on group manifolds and asymmetrically gauged coset spaces [41, 42]. Although there has been some work recently on D-branes in asymmetrically gauged coset theories [43, this subject is still not welldeveloped. Nevertheless there exist several important examples where such backgrounds play a prominent role. Let us only mention at this place the Nappi-Witten background [44, 45] and the $T^{p q}$ spaces [46]. The first of them provides an interesting example of a cosmological background with big-bang and big-crunch singularity while the second is related to the base of a conifold for $p=q=1$ 47, 48].

The relation between string theory on group manifolds and asymmetrically gauged coset spaces may be extended beyond the simple observation of target space reinterpretation. Our results indeed admit an immediate generalization to symmetry breaking D-branes in asymmetric coset models by simply gauging a subgroup of the $H$ symmetry which was preserved by the symmetry breaking D-branes in the group manifold. This is completely obvious from the geometric and the Lagrangian point of view. Gauging a subgroup of $G$ makes necessary the introduction of a gauge field interaction term [49, 50, 42]. The boundary contribution, however, remains unaffected by this extra term. A first step towards a general and comprehensive description of asymmetrically gauged coset models in terms of an algebraic and geometric analysis including D-branes will follow in [28].

To summarize, our results along with those of [20] provide a powerful tool to push forward the programme of classifying D-branes in a given background. It would be very interesting to apply these methods to examples which possess a richer structure of nonabelian subgroups. Let us emphasize the general feature that the dimension of the Dbranes increases with the amount of symmetry breaking we enforce. In particular we have argued that one should generically be able to recover space-filling D-branes. To obtain a complete picture of D-branes in group manifolds and coset models it remains to analyze the stability and the dynamics of symmetry breaking D-branes along the lines of [11, 12] and 15, 27, 51, 14. In this context one would also like to address the question of D-brane charges and K-theory [16, 18. This would require an extension of our results to include supersymmetry.

\section{Acknowledgements}

The author is grateful to A. Yu. Alekseev, S. Fredenhagen, S. Ribault and V. Schomerus for useful discussions and comments on the manuscript. This paper was strongly influ- 
enced by the collaboration with V. Schomerus on asymmetrically gauged coset theories 28]. The present work was financially supported by the Studienstiftung des deutschen Volkes.

\section{A Computational details}

In this appendix we will provide the computational details which have been omitted in section 2. It is convenient to choose a more general framework and to generalize the notion of twisted conjugacy class. We will then be able to include recent proposals of [43] in our description.

In the general approach we start with a family $U_{l}(l=1, \ldots, n)$ of continuous subgroups $H \hookrightarrow U_{l} \hookrightarrow G$ which do not necessarily satisfy the embedding chain property (2.1). To each of these subgroups we associate three embeddings $\epsilon^{H U_{l}}: H \rightarrow U_{l}$ and $\epsilon_{L / R}^{U_{l} G}: U_{l} \rightarrow G$. The indices $L / R$ stand for left and right, respectively. We then define generalized twisted conjugacy classes of $U_{l}$ in $G$ by

$$
\mathcal{C}_{\tilde{f}_{l}}^{U_{l}, G}\left(\epsilon_{L}^{U_{l} G}, \epsilon_{R}^{U_{l} G}\right)=\left\{c_{l}=\epsilon_{L}^{U_{l} G}\left(s_{l}\right) \cdot \tilde{f}_{l} \cdot \epsilon_{R}^{U_{l} G}\left(s_{l}^{-1}\right) \mid s_{l} \in U_{l}\right\} .
$$

These generalized twisted conjugacy classes admit an action of $H$ under which they transform as

$$
s_{l} \mapsto \epsilon^{H U_{l}}(h) \cdot s_{l} \quad \Rightarrow \quad c_{l} \mapsto \epsilon_{L}^{U_{l} G} \circ \epsilon^{H U_{l}}(h) \cdot c_{l} \cdot \epsilon_{R}^{U_{l} G} \circ \epsilon^{H U_{l}}\left(h^{-1}\right) .
$$

By putting $\epsilon_{L}^{U_{l} G}=\epsilon^{U_{l} G}, \epsilon_{R}^{U_{l} G}=\epsilon_{\Omega}^{U_{l} G} \circ \Omega_{l}$ and $\tilde{f}_{l}=\epsilon^{U_{l} G}\left(f_{l}\right)$ we could recover ordinary twisted conjugacy classes in this more general framework, i.e. the setup of section Q. One easily verifies that the set $\mathcal{D}\left\{U_{l}, \epsilon_{L / R}^{U_{l} G}, \tilde{f}_{l}\right\}$ which is generated by the following product of generalized twisted conjugacy classes,

$$
\mathcal{D}\left\{U_{l}, \epsilon_{L / R}^{U_{l} G}, \tilde{f}_{l}\right\}=\mathcal{C}_{\tilde{f}_{n}}^{U_{n}, G}\left(\epsilon_{L}^{U_{n} G}, \epsilon_{R}^{U_{n} G}\right) \cdot \ldots \cdot \mathcal{C}_{\tilde{f}_{1}}^{U_{1}, G}\left(\epsilon_{L}^{U_{1} G}, \epsilon_{R}^{U_{1} G}\right) \subset G,
$$

is invariant under the action of $H$ provided that the embedding maps satisfy the relations

$$
\epsilon_{R}^{U_{l+1} G} \circ \epsilon^{H U_{l+1}}=\epsilon_{L}^{U_{l} G} \circ \epsilon^{H U_{l}} .
$$

For later purposes we also have to demand that the embedding indices of the left and right embeddings $\epsilon_{L / R}^{U_{l} G}$ are identical for fixed subgroup $U_{l}$. Both conditions are automatically satisfied if we restrict to the original setup of section 2. The elements $x \in \mathcal{D}\left\{U_{l}, \epsilon_{L / R}^{U_{l} G}, \tilde{f}_{l}\right\}$ transform under these conditions according to

$$
x \mapsto \epsilon_{L}^{U_{n} G} \circ \epsilon^{H U_{n}}(h) \cdot x \cdot \epsilon_{R}^{U_{1} G} \circ \epsilon^{H U_{1}}\left(h^{-1}\right) .
$$

To write down the boundary WZNW functional for D-branes candidates which are localized along $\mathcal{D}\left\{U_{l}, \epsilon_{L / R}^{U_{l} G}, \tilde{f}_{l}\right\}$ we have to generalize the definition (2.9) to

$$
\omega_{\mathcal{D}}\left(c_{l}\right)=\operatorname{tr}_{R}\left\{\epsilon_{L}^{U_{l} G}\left(s_{l}^{-1} d s_{l}\right) \tilde{f}_{l} \epsilon_{R}^{U_{l} G}\left(s_{l}^{-1} d s_{l}\right) \tilde{f}_{l}^{-1}\right\} .
$$


In this appendix we will present the computational details that a) the boundary WZNW functional (2.7) is invariant under the infinitesimal action (A.1, A.4) of $h=$ $1+i \omega \in H$ on the boundary and b) that it is well-defined with respect to infinitesimal deformations of the disc $D$. We will, however, not be concerned with global issues which give rise to quantization of generalized twisted conjugacy classes and branching selection rules. These global topological properties may possibly lead to severe restrictions which prohibit certain subsets of the form (A.2). For example we would not know how to model maximally symmetric D-branes which are localized along the product of two conjugacy classes $\mathcal{C}_{f_{1}}^{G} \cdot \mathcal{C}_{f_{2}}^{G}$ in the algebraic description. This example suggests that there also might be problems with the conformal invariance of our boundary WZNW functional (2.7) on the quantum level as there is no algebraic description corresponding to this more general setting. These questions have to be addressed in future work.

Let us start with item a), i.e. the invariance of the action functional (2.7) under transformations of the form (A.1, A.4) on the boundary. Referring to the discussion in section 2 this amounts to a proof of the relation $\left.\delta \omega^{\mathrm{WZ}}\right|_{D}=d \delta \omega_{\mathcal{D}}$. The elements of the twisted conjugacy classes transform according to

$$
\delta c_{l}=i \omega_{L}^{(l)} c_{l}-i c_{l} \omega_{R}^{(l)}
$$

where we introduced the short hand notations $\omega_{L / R}^{(l)}=\epsilon_{L / R}^{U_{l} G} \circ \epsilon^{H U_{l}}(\omega)$. The condition (A.3) of mutual consistency of the embedding maps obviously translates into the relation $\omega_{R}^{(l+1)}=\omega_{L}^{(l)}$. Supplied with this information it is now very easy to calculate all variations

$$
\delta\left(c_{l} c_{l-1} \cdots c_{k+1} c_{k}\right)=i \omega_{L}^{(l)} c_{l} c_{l-1} \cdots c_{k+1} c_{k}+i c_{l} c_{l-1} \cdots c_{k+1} c_{k} \omega_{R}^{(k)} .
$$

Similar relations hold for the inverse $\delta c_{l}^{-1}=i \omega_{R}^{(l)} c_{l}^{-1}-i c_{l}^{-1} \omega_{L}^{(l)}$ and for chains of the form $c_{k}^{-1} c_{k+1}^{-1} \cdots c_{l-1}^{-1} c_{l}^{-1}$. Finally, we also need to know the variation

$$
\delta d c_{l}=d\left(i \omega_{L}^{(l)} c_{l}-i c_{l} \omega_{R}^{(l)}\right)=i d \omega_{L}^{(l)} c_{l}-i c_{l} d \omega_{R}^{(l)}+i \omega_{L}^{(l)} d c_{l}-i d c_{l} \omega_{R}^{(l)} .
$$

Due to these relations the transformation properties of $\omega_{\mathcal{D}}\left(c_{k}, \cdots, c_{l}\right)$ may easily be calculated. It turns out that all terms involving $\omega$ cancel each other. Only four terms involving $d \omega$ survive. We summarize this result in

$$
\begin{aligned}
\delta \omega_{\mathcal{D}}\left(c_{k}, \cdots, c_{l}\right)= & -i \operatorname{tr}\left\{c_{k}^{-1} \cdots c_{l}^{-1} d \omega_{L}^{(l)} c_{l} \cdots c_{k+1} d c_{k}\right\} \\
& +i \operatorname{tr}\left\{c_{k}^{-1} \cdots c_{l-1}^{-1} d \omega_{L}^{(l-1)} c_{l-1} \cdots c_{k+1} d c_{k}\right\} \\
& -i \operatorname{tr}\left\{c_{k+1}^{-1} \cdots c_{l}^{-1} d c_{l} c_{l-1} \cdots c_{k+1} d \omega_{R}^{(k+1)}\right\} \\
& +i \operatorname{tr}\left\{c_{k}^{-1} \cdots c_{l}^{-1} d c_{l} c_{l-1} \cdots c_{k} d \omega_{R}^{(k)}\right\} .
\end{aligned}
$$

When evaluating this expression special care has to be taken if $l=k+1$. In this case no factors $c_{l-1} \cdots c_{k+1}$ appear between the differentials in lines two and three. 
Due to its different structure the variation of $\omega_{\mathcal{D}}\left(c_{l}\right)$ has to be treated separately. In this case we obtain

$$
\delta \omega_{\mathcal{D}}\left(c_{l}\right)=-i \operatorname{tr}\left\{d \omega_{L}^{(l)} d c_{l} c_{l}^{-1}+d \omega_{R}^{(l)} c_{l}^{-1} d c_{l}\right\}
$$

During the calculation we made use of

$$
\begin{aligned}
& c_{l}^{-1} d c_{l}=\epsilon_{R}^{U_{l} G}\left(s_{l}\right) \tilde{f}_{l}^{-1} \epsilon_{L}^{U_{l} G}\left(s_{l}^{-1} d s_{l}\right) \tilde{f}_{l} \epsilon_{R}^{U_{l} G}\left(s_{l}^{-1}\right)-\epsilon_{R}^{U_{l} G}\left(d s_{l} s_{l}^{-1}\right) \\
& d c_{l} c_{l}^{-1}=\epsilon_{L}^{U_{l} G}\left(d s_{l} s_{l}^{-1}\right)-\epsilon_{L}^{U_{l} G}\left(s_{l}\right) \tilde{f}_{l} \epsilon_{R}^{U_{l} G}\left(s_{l}^{-1} d s_{l}\right) \tilde{f}_{l}^{-1} \epsilon_{L}^{U_{l} G}\left(s_{l}^{-1}\right) .
\end{aligned}
$$

Indeed, these two relations imply

$$
i \operatorname{tr}\left\{d \omega_{L}^{(l)} d c_{l} c_{l}^{-1}+d \omega_{R}^{(l)} c_{l}^{-1} d c_{l}\right\}=-\delta \omega_{\mathcal{D}}\left(c_{l}\right)+i \operatorname{tr}\left\{d \omega_{L}^{(l)} \epsilon_{L}^{U_{l} G}\left(d s_{l} s_{l}^{-1}\right)-d \omega_{R}^{(l)} \epsilon_{R}^{U_{l} G}\left(d s_{l} s_{l}^{-1}\right)\right\} .
$$

If we rewrite the last term according to

$$
\operatorname{tr}\left\{\epsilon_{L}^{U_{l} G}\left(\epsilon^{H U_{l}}(d \omega) d s_{l} s_{l}^{-1}\right)-\epsilon_{R}^{U_{l} G}\left(\epsilon^{H U_{l}}(d \omega) d s_{l} s_{l}^{-1}\right)\right\}
$$

we see that it vanishes provided the two embeddings $\epsilon_{L / R}^{U_{l} G}$ have the same embedding index.

Summing up all contributions and remembering that the variation of $\omega_{\mathcal{D}}\left(c_{k}, c_{k+1}\right)$ shows some subtleties we obtain

$$
\delta \omega_{\mathcal{D}}=-i \sum_{l=1}^{n} \operatorname{tr}\left\{d \omega_{L}^{(n)} c_{n} \cdots c_{l+1} d c_{l} c_{l}^{-1} \cdots c_{n}^{-1}+d \omega_{R}^{(1)} c_{1}^{-1} \cdots c_{l}^{-1} d c_{l} c_{l-1} \cdots c_{1}\right\}
$$

During the calculation we made use of several cancellations. Finally, we have to compare this expression with the variation of the Wess-Zumino term. A careful calculation gives

$$
\delta \omega^{\mathrm{WZ}}=-i d \operatorname{tr}\left\{d \omega_{L}^{(n)} d g g^{-1}+d \omega_{R}^{(1)} g^{-1} d g\right\}
$$

This may easily be evaluated using the relations

$$
g^{-1} d g=\sum_{l=1}^{n} c_{1}^{-1} \cdots c_{l}^{-1} d c_{l} c_{l-1} \cdots c_{1}, \quad \quad d g g^{-1}=\sum_{l=1}^{n} c_{n} \cdots c_{l+1} d c_{l} c_{l}^{-1} \cdots c_{n}^{-1}
$$

The variation then reads

$$
\delta \omega^{\mathrm{WZ}}=-i \sum_{l=1}^{n} d \operatorname{tr}\left\{d \omega_{L}^{(n)} c_{n} \cdots c_{l+1} d c_{l} c_{l}^{-1} \cdots c_{n}^{-1}+d \omega_{R}^{(1)} c_{1}^{-1} \cdots c_{l}^{-1} d c_{l} c_{l-1} \cdots c_{1}\right\}
$$


Obviously, the contributions from $\delta \omega^{\mathrm{WZ}}$ and $\delta \omega_{\mathcal{D}}$ cancel each other exactly. This proves that the product of generalized twisted conjugacy classes is indeed a valid candidate for the geometry of D-branes which preserve an action of the group $H$.

Now we are able to address item b), i.e. the invariance of the action functional (2.7) under infinitesimal deformations of the disc $D$. It is sufficient to proof the relation $d \omega_{\mathcal{D}}=$ $\left.\omega^{\mathrm{WZ}}\right|_{D}$. The calculation turns out to be very involved if one tries to perform it directly. It is convenient to use an induction argument instead, i.e. we supply the boundary twoform with an additional label $n$ and write $\omega_{\mathcal{D}}(n)$. The number $n$ ist just the number of generalized twisted conjugacy classes appearing in eq. (A.2). For $n=1$ we have $\omega_{\mathcal{D}}(1)=\omega_{\mathcal{D}}\left(c_{1}\right)$. Let us thus first determine

$$
\begin{aligned}
d \omega_{\mathcal{D}}\left(c_{l}\right)= & -\operatorname{tr}\left\{\epsilon_{L}^{U_{l} G}\left(s_{l}^{-1} d s_{l} s_{l}^{-1} d s_{l}\right) \tilde{f}_{l} \epsilon_{R}^{U_{l} G}\left(s_{l}^{-1} d s_{l}\right) \tilde{f}_{l}^{-1}\right\} \\
& +\operatorname{tr}\left\{\epsilon_{L}^{U_{l} G}\left(s_{l}^{-1} d s_{l}\right) \tilde{f}_{l} \epsilon_{R}^{U_{l} G}\left(s_{l}^{-1} d s_{l} s_{l}^{-1} d s_{l}\right) \tilde{f}_{l}^{-1}\right\} .
\end{aligned}
$$

On the other hand we have

$$
\begin{aligned}
\omega^{\mathrm{WZ}}\left(c_{l}\right)= & \frac{1}{3} \operatorname{tr}\left\{\left(\epsilon_{R}^{U_{l} G}\left(s_{l}\right) \tilde{f}_{l}^{-1} \epsilon_{L}^{U_{l} G}\left(s_{l}^{-1} d s_{l}\right) \tilde{f}_{l} \epsilon_{R}^{U_{l} G}\left(s_{l}^{-1}\right)-\epsilon_{R}^{U_{l} G}\left(d s_{l} s_{l}^{-1}\right)\right)^{3}\right\} \\
= & \frac{1}{3} \operatorname{tr}\left\{\epsilon_{L}^{U_{l} G}\left(\left(s_{l}^{-1} d s_{l}\right)^{3}\right)-\epsilon_{R}^{U_{l} G}\left(\left(d s_{l} s_{l}^{-1}\right)^{3}\right)\right\} \\
& -\operatorname{tr}\left\{\tilde{f}_{l}^{-1} \epsilon_{L}^{U_{l} G}\left(s_{l}^{-1} d s_{l} s_{l}^{-1} d s_{l}\right) \tilde{f}_{l} \epsilon_{R}^{U_{l} G}\left(s_{l}^{-1} d s_{l}\right)\right\} \\
& +\operatorname{tr}\left\{\tilde{f}_{l}^{-1} \epsilon_{L}^{U_{l} G}\left(s_{l}^{-1} d s_{l}\right) \tilde{f}_{l} \epsilon_{R}^{U_{l} G}\left(s_{l}^{-1} d s_{l} s_{l}^{-1} d s_{l}\right)\right\} .
\end{aligned}
$$

The first two terms vanish as the two embeddings by assumption have the same embedding index. By specializing to $l=1$ we have proven $d \omega_{\mathcal{D}}(n)=\left.\omega^{\mathrm{WZ}}\right|_{D}$ for $n=1$.

Let us now turn to the case $n>1$. It is convenient to introduce the notation $g_{n}=$ $c_{n} \cdots c_{1}=c_{n} g_{n-1}$. In addition we also need the recursion property $\omega_{\mathcal{D}}(n)=\omega_{\mathcal{D}}(n-$ 1) $+\sum_{l=1}^{n} \omega_{\mathcal{D}}\left(c_{l}, \cdots, c_{n}\right)$. Using the representation above we easily obtain $g_{n}^{-1} d g_{n}=$ $g_{n-1}^{-1} c_{n}^{-1} d c_{n} g_{n-1}+g_{n-1}^{-1} d g_{n-1}$. We are thus able to calculate

$$
\begin{aligned}
\omega^{\mathrm{WZ}}\left(g_{n}\right)= & \omega^{\mathrm{WZ}}\left(g_{n-1}\right)+\omega^{\mathrm{WZ}}\left(c_{l}\right) \\
& +\operatorname{tr}\left\{c_{n}^{-1} d c_{n} c_{n}^{-1} d c_{n} d g_{n-1} g_{n-1}^{-1}+c_{n}^{-1} d c_{n} d g_{n-1} g_{n-1}^{-1} d g_{n-1} g_{n-1}^{-1}\right\} .
\end{aligned}
$$

By induction we have $d \omega_{\mathcal{D}}(n-1)=\omega^{\mathrm{WZ}}\left(g_{n-1}\right)$. We also proved already that $d \omega\left(c_{n}\right)=$ $\omega^{\mathrm{WZ}}\left(c_{n}\right)$. It thus remains to check whether

$$
\sum_{l=1}^{n-1} d \omega_{\mathcal{D}}\left(c_{l}, \cdots, c_{n}\right)=\operatorname{tr}\left\{c_{n}^{-1} d c_{n} c_{n}^{-1} d c_{n} d g_{n-1} g_{n-1}^{-1}+c_{n}^{-1} d c_{n} d g_{n-1} g_{n-1}^{-1} d g_{n-1} g_{n-1}^{-1}\right\}
$$


Indeed, for the left hand side we have

$$
\begin{aligned}
\sum_{l=1}^{n-1} d \omega_{\mathcal{D}}\left(c_{l}, \cdots, c_{n}\right) & =\sum_{l=1}^{n-1} \sum_{k=l}^{n-1} \operatorname{tr}\left\{c_{l}^{-1} \cdots c_{k}^{-1} d c_{k} c_{k}^{-1} \cdots c_{n}^{-1} d c_{n} c_{n-1} \cdots c_{l+1} d c_{l}\right\} \\
& +\sum_{l=1}^{n-1} \operatorname{tr}\left\{c_{l}^{-1} \cdots c_{n}^{-1} d c_{n} c_{n}^{-1} d c_{n} c_{n-1} \cdots c_{l+1} d c_{l}\right\} \\
& +\sum_{l=1}^{n-1} \sum_{k=l+1}^{n-1} \operatorname{tr}\left\{c_{l}^{-1} \cdots c_{n}^{-1} d c_{n} c_{n-1} \cdots c_{k+1} d c_{k} c_{k-1} \cdots c_{l+1} d c_{l}\right\}
\end{aligned}
$$

To evaluate the right hand side of eq. (A.5) we use the explicit form of $g_{n-1}$ as a product of $c$ 's and write

$$
\begin{aligned}
g_{n-1}^{-1} d g_{n-1} & =\sum_{l=1}^{n-1} c_{1}^{-1} \cdots c_{l}^{-1} d c_{l} c_{l-1} \cdots c_{1} \\
d g_{n-1} g_{n-1}^{-1} & =\sum_{l=1}^{n-1} c_{n-1} \cdots c_{l+1} d c_{l} c_{l}^{-1} \cdots c_{n-1}^{-1} .
\end{aligned}
$$

Taking the square of the last expression we arrive at

$$
\begin{aligned}
d g_{n-1} g_{n-1}^{-1} d g_{n-1} g_{n-1}^{-1}= & \sum_{l=1}^{n-1} \sum_{k=1}^{n-1} c_{n-1} \cdots c_{l+1} d c_{l} c_{l}^{-1} \cdots c_{n-1}^{-1} c_{n-1} \cdots c_{k+1} d c_{k} c_{k}^{-1} \cdots c_{n-1}^{-1} \\
= & \sum_{l=1}^{n-1} \sum_{k=l}^{n-1} c_{n-1} \cdots c_{l+1} d c_{l} c_{l}^{-1} \cdots c_{k}^{-1} d c_{k} c_{k}^{-1} \cdots c_{n-1}^{-1} \\
& +\sum_{l=1}^{n-1} \sum_{k=l+1}^{n-1} c_{n-1} \cdots c_{k+1} d c_{k} c_{k-1} \cdots c_{l+1} d c_{l} c_{l}^{-1} \cdots c_{n-1}^{-1} .
\end{aligned}
$$

Plugging all this into the right hand side of eq. (A.5) we finally arrive at

$$
\begin{aligned}
& \operatorname{tr}\left\{c_{n}^{-1} d c_{n} c_{n}^{-1} d c_{n} d g_{n-1} g_{n-1}^{-1}+c_{n}^{-1} d c_{n} d g_{n-1} g_{n-1}^{-1} d g_{n-1} g_{n-1}^{-1}\right\} \\
= & \sum_{l=1}^{n-1} \operatorname{tr}\left\{c_{n}^{-1} d c_{n} c_{n}^{-1} d c_{n} c_{n-1} \cdots c_{l+1} d c_{l} c_{l}^{-1} \cdots c_{n-1}^{-1}\right\} \\
& +\sum_{l=1}^{n-1} \sum_{k=l}^{n-1} \operatorname{tr}\left\{c_{n}^{-1} d c_{n} c_{n-1} \cdots c_{l+1} d c_{l} c_{l}^{-1} \cdots c_{k}^{-1} d c_{k} c_{k}^{-1} \cdots c_{n-1}^{-1}\right\} \\
& +\sum_{l=1}^{n-1} \sum_{k=l+1}^{n-1} \operatorname{tr}\left\{c_{n}^{-1} d c_{n} c_{n-1} \cdots c_{k+1} d c_{k} c_{k-1} \cdots c_{l+1} d c_{l} c_{l}^{-1} \cdots c_{n-1}^{-1}\right\}
\end{aligned}
$$

This expression coincides with the expression (A.6). We have thus completed our induction argument. 


\section{References}

[1] E. Witten, Nonabelian bosonization in two dimensions, Commun. Math. Phys. 92 (1984) 455-472.

[2] V. G. Knizhnik and A. B. Zamolodchikov, Current algebra and Wess-Zumino model in two dimensions, Nucl. Phys. B247 (1984) 83-103.

[3] D. Gepner and E. Witten, String theory on group manifolds, Nucl. Phys. B278 (1986) 493.

[4] P. Goddard, A. Kent and D. I. Olive, Virasoro algebras and coset space models, Phys. Lett. B152 (1985) 88.

[5] J. L. Cardy, Boundary conditions, fusion rules and the Verlinde formula, Nucl. Phys. B324 (1989) 581.

[6] L. Birke, J. Fuchs and C. Schweigert, Symmetry breaking boundary conditions and WZW orbifolds, Adv. Theor. Math. Phys. 3 (1999) 671-726 hep-th/9905038].

[7] R. E. Behrend, P. A. Pearce, V. B. Petkova and J.-B. Zuber, Boundary conditions in rational conformal field theories, Nucl. Phys. B570 (2000) 525-589 hep-th/9908036.

[8] A. Yu. Alekseev and V. Schomerus, D-branes in the WZW model, Phys. Rev. D60 (1999) 061901 hep-th/9812193.

[9] G. Felder, J. Fröhlich, J. Fuchs and C. Schweigert, The geometry of WZW branes, J. Geom. Phys. 34 (2000) 162-190 hep-th/9909030.

[10] K. Gawedzki, Conformal field theory: A case study, hep-th/9904145.

[11] C. Bachas, M. R. Douglas and C. Schweigert, Flux stabilization of D-branes, JHEP 05 (2000) 048 hep-th/0003037.

[12] P. Bordalo, S. Ribault and C. Schweigert, Flux stabilization in compact groups, JHEP 10 (2001) 036 hep-th/0108201.

[13] A. Yu. Alekseev, A. Recknagel and V. Schomerus, Non-commutative world-volume geometries: Branes on SU(2) and fuzzy spheres, JHEP 09 (1999) 023 hep-th/9908040.

[14] A. Yu. Alekseev, S. Fredenhagen, T. Quella and V. Schomerus, Non-commutative gauge theory of twisted D-branes, hep-th/0205123. To appear in Nucl. Phys. B.

[15] A. Yu. Alekseev, A. Recknagel and V. Schomerus, Brane dynamics in background fluxes and non-commutative geometry, JHEP 05 (2000) 010 hep-th/0003187. 
[16] S. Fredenhagen and V. Schomerus, Branes on group manifolds, gluon condensates, and twisted K-theory, JHEP 04 (2001) 007 hep-th/0012164.

[17] J. Maldacena, G. W. Moore and N. Seiberg, Geometrical interpretation of D-branes in gauged WZW models, JHEP 07 (2001) 046 hep-th/0105038.

[18] J. Maldacena, G. W. Moore and N. Seiberg, D-brane instantons and K-theory charges, JHEP 11 (2001) 062 hep-th/0108100.

[19] M. R. Gaberdiel, A. Recknagel and G. M. T. Watts, The conformal boundary states for SU(2) at level 1, Nucl. Phys. B626 (2002) 344-362 hep-th/0108102.

[20] T. Quella and V. Schomerus, Symmetry breaking boundary states and defect lines, JHEP 06 (2002) 028 hep-th/0203161.

[21] G. Sarkissian, Non-maximally symmetric D-branes on group manifold in the Lagrangian approach, JHEP 07 (2002) 033 hep-th/0205097.

[22] S. Stanciu, D-branes in an AdS(3) background, JHEP 09 (1999) 028 hep-th/9901122.

[23] C. Bachas and M. Petropoulos, Anti-de-Sitter D-branes, JHEP 02 (2001) 025 hep-th/0012234.

[24] A. Rajaraman, New AdS(3) branes and boundary states, hep-th/0109200.

[25] K. Gawedzki, Boundary WZW, G/H, G/G and CS theories, hep-th/0108044.

[26] S. Elitzur and G. Sarkissian, D-branes on a gauged WZW model, Nucl. Phys. B625 (2002) 166-178 hep-th/0108142].

[27] S. Fredenhagen and V. Schomerus, D-branes in coset models, JHEP 02 (2002) 005 hep-th/0111189.

[28] T. Quella and V. Schomerus. To appear.

[29] A. Recknagel and V. Schomerus, D-branes in Gepner models, Nucl. Phys. B531 (1998) 185-225 [hep-th/9712186].

[30] J. Fuchs and C. Schweigert, Branes: From free fields to general backgrounds, Nucl. Phys. B530 (1998) 99-136 hep-th/9712257.

[31] J. Fuchs and C. Schweigert, Symmetry breaking boundaries. I: General theory, Nucl. Phys. B558 (1999) 419 [hep-th/9902132].

[32] J. Fuchs and C. Schweigert, Symmetry breaking boundaries. II: More structures, examples, Nucl. Phys. B568 (2000) 543 hep-th/9908025. 
[33] J. Fuchs, I. Runkel and C. Schweigert, TFT construction of RCFT correlators. I: Partition functions, hep-th/0204148.

[34] H. Ishikawa and T. Tani, Novel construction of boundary states in coset conformal field theories, hep-th/0207177.

[35] A. Recknagel, Permutation branes, hep-th/0208119.

[36] J. M. Figueroa-O'Farrill and S. Stanciu, D-branes in $A d S(3) \times S(3) \times S(3) \times S(1)$, JHEP 04 (2000) 005 hep-th/0001199.

[37] A. Karch and L. Randall, Open and closed string interpretation of SUSY CFT's on branes with boundaries, JHEP 06 (2001) 063 [hep-th/0105132].

[38] O. DeWolfe, D. Z. Freedman and H. Ooguri, Holography and defect conformal field theories, Phys. Rev. D66 (2002) 025009 [hep-th/0111135.

[39] C. Bachas, J. de Boer, R. Dijkgraaf and H. Ooguri, Permeable conformal walls and holography, JHEP 06 (2002) 027 hep-th/0111210.

[40] J. Erdmenger, Z. Guralnik and I. Kirsch, Four-dimensional superconformal theories with interacting boundaries or defects, Phys. Rev. D66 (2002) 025020 hep-th/0203020.

[41] E. Guadagnini, M. Martellini and M. Mintchev, Scale invariant sigma models on homogeneous spaces, Phys. Lett. B194 (1987) 69.

[42] I. Bars and K. Sfetsos, Generalized duality and singular strings in higher dimensions, Mod. Phys. Lett. A7 (1992) 1091-1104 hep-th/9110054.

[43] M. A. Walton and J.-G. Zhou, D-branes in asymmetrically gauged WZW models and axial-vector duality, hep-th/0205161.

[44] C. R. Nappi and E. Witten, A closed, expanding universe in string theory, Phys. Lett. B293 (1992) 309-314 [hep-th/9206078].

[45] S. Elitzur, A. Giveon, D. Kutasov and E. Rabinovici, From big bang to big crunch and beyond, JHEP 06 (2002) 017 hep-th/0204189].

[46] L. A. Pando-Zayas and A. A. Tseytlin, Conformal sigma models for a class of T(p,q) spaces, Class. Quant. Grav. 17 (2000) 5125-5131 hep-th/0007086.

[47] P. Candelas and X. C. de la Ossa, Comments on conifolds, Nucl. Phys. B342 (1990) 246-268.

[48] I. R. Klebanov and E. Witten, Superconformal field theory on three-branes at a Calabi-Yau singularity, Nucl. Phys. B536 (1998) 199-218 [hep-th/9807080]. 
[49] K. Gawedzki and A. Kupiainen, G/H conformal field theory from gauged WZW model, Phys. Lett. B215 (1988) 119-123.

[50] D. Karabali, Q.-H. Park, H. J. Schnitzer and Z. Yang, A GKO construction based on a path integral formulation of gauged Wess-Zumino-Witten actions, Phys. Lett. B216 (1989) 307.

[51] S. Fredenhagen and V. Schomerus, On boundary RG-flows in coset conformal field theories, hep-th/0205011. 